\title{
A Perturbation Method for Studying Thermal Convection Problems
}

\author{
By \\ Bin OKaI \\ Geophysical Institute, Faculty of Science, Tokyo University, Tolyo
}

\begin{abstract}
When a layer of fluid is heated uniformly from below, and the Rayleigh number exceeds a critical value, convection takes place in it in a regular cellular pattern. A perturbation method is presented here for determining mathematically the pattern and amplitude of this steady convective motion. The essential point of the method is to expand functions which describe the velocity and temperature field in the fluid in a power series of a parameter $\varepsilon$, while the Rayleigh number is put as a product of the critical value times $\left(1+-\varepsilon^{2}\right)$. A set of inhomogeneous equations thus obtained can be solved by the perturbation method which is in use in non-linear oscillation problems. In the twodimensional case, the slope of heat transport curve becomes steepened abruptly when the Rayleigh numher exceeds a critical value. Another problem which can be dealt with by this method is that of convection within a sphere. This forms an extension of CHANDRASEKHAR's linearized stability theory.

Furthermore, a study is made of the steady thermal convection in a two-dimensional fluid layer when it is heated uniformly from below under a simultaneous constraint of nonuniform temperature given on its upper surface. Mathematically this is an application of the method mentioned above to a problem with inhomogeneous boundary conditions. It was found that the site of spontaneous convection cells is governed by the surface temperature disturbance having a critical wave length. Surface temperature disturbances having much longer or shorter wave lengths play very little part in this, while those having wave lengths close to the critical one have influential effect in determining the general feature of fluid motion.
\end{abstract}

\$1. Although the earth's mantle behaves as a solid for forces of short duration such as those occurring in earthquake waves and in earth tides, it may well behave as a fluid for those of very long cluration, such as suggested by approximate isostatic adjustments of large topographic features. If this is actually so, there is a possibility that the mantle may be subject to a very slow convection (Perkers, 1935; Hatrss, 1936; Bullakd, 195()). Of course, some difficulties arise in supposing convection currents in the mantle. Brich (1951) has pointed out that the currents, if they take place at all, can hardly extend up to the $20^{\circ}$ discontinuity. Two recent discoveries appear, however, to support the hypothesis of convection in the mantle. One is concerned with the heat flow from within the earth. The measurements which have been made up to now in this connection (Bullart), 1954; BuL- lard, Maxwell and Revelde, 1956; Herzen, 1959) appear to inclicate that the heat flow in oceanic bottoms does not differ significantly from that in continents. The source of heat is reasonably supposed to be due to radioactivity within the crust, which is $35 \mathrm{~km}$ thick on the continent and $5 \mathrm{~km}$ under the ocean. Where does the oceanic heat come from? If radioactivity is distributed through too great a depth, the temperature near the bottom of the radioactive layer will rise above its melt. ing point, and this is inconsistent with the fact that $S$ waves can be propagated at these depths. This difficulty may be avoided if the transport of heat by convection in the mantle is assumed. The other discovery is concerned with palaeomagnetism. Studies of palaeomag. netism (Blackett, 1956; Runcorn, 1955; Creer, Irving and Runcorn, 1954) have rejuvenated interest about the classical hypo- 
thesis of continental drifts. But some reasonable forces which drive continental blocks in motion must be looked for if the hypothesis is to become more than a mere hypothesis. Here again, these forces may be assumed to be due to convection currents within the mantle.

Although there are evidences like these in favour of convection currents in the mantle, nothing definite can be said at present about the existence of such large scale motions itself. This is partly because we do not have enough knowledge about the earth and partly because mathematical studies of convection so far developed are far from being satisfactory for the purpose. There are many fundamental problems of thermal convection to be solved. How much heat is transported after convection once takes place in a layer of fluid which so far has been at rest? What would the distribution of temperature become in a convective layer? In 1958, MALKUS and VeronIS presented a perturbation method by which the form and amplitude of cellular convection can be determined. Their intention was to complete RAYLEIGH's first-order solution of the problem. At about the same time the present writer found a similar method independently. It was when he had finished his calculations up to $\S 8$ of the present article, that he was informed of MaLKUS and Veronis' paper by Dr. Chandrasemerar of Chicago University (Oct. 24, 1958). A part of the results presented here is similar to those of MALKuS and VERONIS. In the following, stress will be laid upon the writer's own method of analysis which is more or less different from theirs.

In $\S 2$, the fundamental equations and boundary conditions of the problem are stated. In $\S 3$, the perturbation method of solutions for the finite amplitude fields of temperature and velocity is described. The method can be applied to other problems, of which a simple example is given. In $\$ 4$, this method is applied to a two-dimensional convection problem (roll case). In \$5, the relation between the heat transport and the mean temperature gradient is studied. In $\S 6$, the fundamental equations and boundary conditions are describ- ed for the convection within a sphere and in $\$ 7$, analysis is carried out on this problem. In $\S 8$, convective fluid motions which take place within a slowly rotating sphere are considered. Up to this point the boundary conditions are homogeneous. Hereafter inhomogeneous boundary conditions are dealt with. The reason why the inhomogeneity is brought up is as follows. When the temperature difference between the upper and lower surfaces exceeds a certain critical value, convection takes place in a regular cellular pattern, but theory does not distinguish whether horizontal pattern of convection cells is rectangular, triangular or hexagonal. Thus we cannot answer a question such as; Why does one of these patterns take place preferentially, when more than one solution is possible at the point of instability? Even when the motion is taken to be two-dimensional, one cannot predict where the site of upwelling will be located in the fluid. The motion could be upward anywhere, so long as the fluid is uniform horizontally. But such a perfect uniformity cannot be imagined in nature. It is of importance therefore to know the influence of inhomogeneity. Besides, in the case of convection within the earth's mantle, consideration must be paid to the earth's crust of non-uniform thickness. $\$ 9$ and $\S 10$ correspond to $\$ 2$ and $\$ 4$ for homogeneous case respectively. In $\$ 11$ the results obtained are given.

Finally it is admitted that there are many related geophysical problems out of the scope of the new method presented here. This is merely one step toward generalisation of the classical linear stability theory.

§2. First of all, a two-dimensional steady convective motion in a fluid as shown schematically in Fig. 1 will be dealt with. The fluid is supposed to be enclosed between two horizontal conducting surfaces, which are $h$ apart. The lower surface is kept at a temperature $T_{0}$ and the upper surface at a lower temperature $\left(T_{0}-\beta h\right)$, the mean temperature gradient being $-\beta,(\beta>0)$. The fundamental equations are the equation of continuity (1), the equation of motions of NAviER-STOKES (2), (3), the equation of thermal conduction (4) 


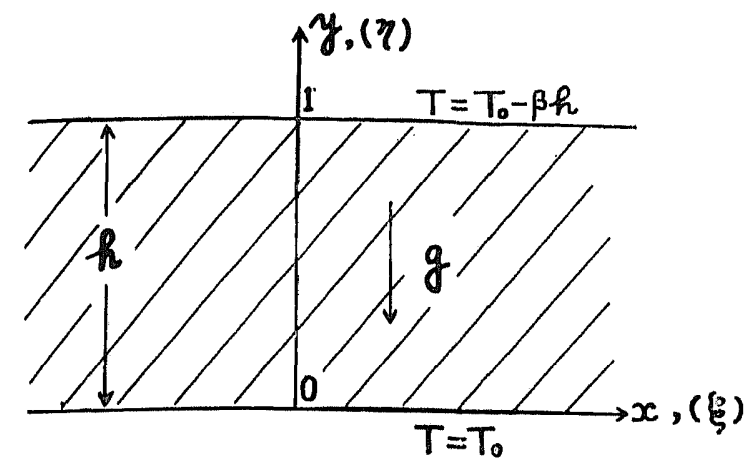

Fig. 1. Fluid layer heated from below. and the equation of thermal expansion (5):

$$
\begin{aligned}
& \frac{\partial u}{\partial x}+\frac{\partial v}{\partial y}=0 \\
& \rho\left(u \frac{\partial u}{\partial x}+v \frac{\partial u}{\partial y}\right)=-\frac{\partial p}{\partial x}+\mu \nabla^{2} u \\
& \rho\left(u \frac{\partial v}{\partial x}+v \frac{\partial v}{\partial y}\right)=-\frac{\partial p}{\partial y}-\rho g+\mu \nabla^{2} v \\
& u \quad \frac{\partial T}{\partial x}+v \frac{\partial T}{\partial y}=\kappa \nabla^{2} T \\
& \rho=\rho_{0}\left\{1-\alpha\left(T-T_{0}\right)\right\},
\end{aligned}
$$

where $u$ and $v$ are the components of fluid velocity in the direction of $x$ and $y$ respectively, $T$ is the temperature, $p$ is the pressure, $g$ is the magnitude of the gravitational acceleration, $\mu$ is the viscosity of the fluid, $\kappa$ is the thermometric conductivity, $\rho$ is the density, and $\alpha$ the linear coefficient of thermal expansion. Using the stream function $\phi, u$ and $v$ may be rewritten as $u=\frac{\partial \phi}{\partial y}$ and $v=-\frac{\partial \phi}{\partial x}$.

At this point it is of value to non-dimensionalize the physical quantities appearing in the equations. This will simplify subsequent mathematical manipulations and help us getting pertinent physical parameters for the problem.

Let $T=\beta h(-y+\tau), x=h \xi, y=h \eta, \phi=\kappa \psi$,

and after these transformations, all primes will be dropped out. Then (2) and (3) are cross-differentiated and the difference taken in order to have pressure eliminated. The resulting relations between $\psi$ and $\tau$ are

$$
\begin{gathered}
\frac{\kappa}{\nu}\left[\frac{\partial \psi}{\partial \eta}\left(\frac{\partial^{3} \psi}{\partial \xi \partial \eta^{2}}+\frac{\partial^{3} \psi}{\partial \xi^{3}}\right)-\frac{\partial \psi}{\partial \xi}\left(\frac{\partial^{3} \psi}{\partial \eta^{3}}+\frac{\partial^{3} \psi}{\partial \xi^{2} \partial \eta}\right)\right] \\
=-R \frac{\partial \tau}{\partial \xi}+\nabla^{4} \psi \\
\frac{\partial \psi}{\partial \eta} \frac{\partial \tau}{\partial \xi}-\frac{\partial \psi}{\partial \xi} \frac{\partial \tau}{\partial \eta}+\frac{\partial \psi}{\partial \xi}=\nabla^{2} \tau .
\end{gathered}
$$

$R \equiv \frac{\alpha \beta g h^{4}}{\kappa \nu}$ is a non-dimensional parameter which is called the RAYLEIGH number and $\frac{\nu}{\kappa}$ is the Prandil number. So far all the non-linear terms have been retained, but, hereafter, for the sake of simplicity, the inertia terms will be ignored, as it is those non-linear terms appearing in (8) that are more essential in the problem. Also, in the case of convection within the earth's mantle, the inertia terms are negligible because the pertaking viscosity is enormously high. Thus the final equations become

$$
\begin{gathered}
\frac{\partial \psi}{\partial y} \frac{\partial \tau}{\partial x}-\frac{\partial \psi}{\partial x} \frac{\partial \tau}{\partial y}+\frac{\partial \psi}{\partial x}=\nabla^{2} \tau \\
\nabla^{4} \psi=R \frac{\partial \tau}{\partial x} .
\end{gathered}
$$

The conditions at the boundaries $(y=1, y=0)$ are

$$
\frac{\partial \psi}{\partial x}=0, \quad \frac{\partial^{2} \psi}{\partial y^{2}}-\frac{\partial^{2} \psi}{\partial x^{2}}=0, \tau=0
$$

following RaYlergh. This means that temperatures are kept constant, the vertical component of velocity vanishes and stress does not exist at the boundaries. In other words, the boundaries are free. More realistic are of course the conditions of 'rigid-rigid' or 'rigid-free', but these are difficult to be taken into account. Essential points of the problem are not lost by adopting the above-mentioned unrealistic conditions.

§3. A convective motion takes place in the fluid layer when the mean temperature gradient $\beta$ (or $R$ ) reaches a critical value $\beta_{0}$ (or $R_{0}$ ). A further increase of mean temperature gradient produces more intense field of motion. Therefore functions $\psi$ and $\tau$ describing the 
field must appear suddenly when $\beta$ reaches $\beta_{0}$. In other words, $\psi$ and $\tau$ are not analytic at $\beta=\beta_{0}$ (Fig. 2). Consequently one cannot expect solutions in the form

$$
\left.\begin{array}{l}
\beta=\beta_{0}(1+\delta) \\
\psi=\delta \cdot \psi_{1}+\delta^{2} \cdot \psi_{2}+\delta^{3} \cdot \psi_{3}+\cdots \\
\tau=\delta \cdot \tau_{1}+\delta^{2} \cdot \tau_{2}+\delta^{3} \cdot \tau_{3}+\cdots
\end{array}\right\}
$$

which are all analytic. If $\psi$ and $\tau$ could be written in the form mentioned above, $\psi$ and $\tau$ could be extended to include the state having the temperature gradient less than the critical one, and this will mean that a convective motion could occur even before the mean temperature gradient $\beta$ reaches the critical value $\beta_{0}$. This is absurd and does not represent actual cases. Therefore another form of solutions has to be looked for. The weakest point of the expression $\beta=\beta_{0}(1+\delta)$ in (12) is that $\beta$ can easily become less than $\beta_{0}$, while physically $\delta$ must always be positive. This can be avoided if $\delta$ is put to be equal to $\varepsilon^{2}$. But if all the $\delta$ 's in (12) are made equal to $\varepsilon^{2}$, the situation will still be the same, for there is no reason to suppose that $\varepsilon$ does not

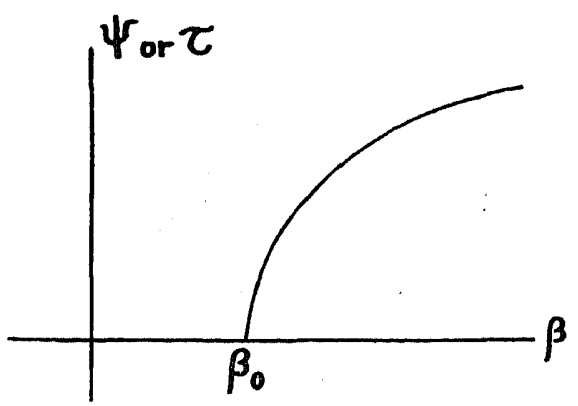

Fig. 2. Relation between mean temp. grad. and the function describing the field. $\psi,(\tau)$ is not analytic at the point where $\beta=\beta_{0}$.

become imaginary and the absurdity remains. The difficulties will disappear if one could find solutions in the following form

$$
\left.\begin{array}{l}
\beta=\beta_{0}\left(1+\varepsilon^{2}\right) \\
\phi=\varepsilon \psi_{1}+\varepsilon^{2} \psi_{2}+\varepsilon^{3} \psi_{3}+\cdots \\
\tau=\varepsilon \tau_{1}+\varepsilon^{2} \tau_{2}+\varepsilon^{3} \tau_{3}+\cdots
\end{array}\right\} .
$$

Of course, here again $\beta$ becomes less than $\beta_{0}$ if $\varepsilon$ is taken as pure imaginary. But $\psi$ and $\tau$, then, become complex functions which have no physical counterpart in reality. This means that $\psi$ and $\tau$ as given in (13) are good enough for describing the field. It is now required that expressions (13) satisfy the equations of motion, (9) and (10), for all values of $\varepsilon$ less than some maximum $\varepsilon_{n}$. The coefficients of each power of $\varepsilon$ obtained on inserting the expressions (13) into the fundamental equations (9), (10) must vanish individually and the resulting series for $\psi$ and $\tau$ must converge if relations (13) are to represent a satisfactory solution to the problem.

The sequence of linear inhomogeneous equations in powers of $\varepsilon$ obtained on inserting relations (13) into equations (9) and (10) is

$$
\begin{gathered}
\Delta^{4} \sum_{i} \varepsilon^{i} \psi_{i}=R_{0}\left(1+\varepsilon^{2}\right)-\frac{\partial}{\partial x} \sum_{i} \varepsilon^{i} \tau_{i} \\
-\frac{\partial}{\partial y} \sum_{i} \varepsilon^{i} \psi_{i}-\frac{\partial}{\partial x} \sum_{i} \varepsilon^{i} \tau_{i}-\frac{\partial}{\partial x} \sum_{i} \varepsilon^{i} \psi_{i}-\frac{\partial}{\partial y} \sum_{i} \varepsilon^{i} \tau_{i} \\
+\frac{\partial}{\partial x} \sum_{i} \varepsilon^{i} \psi_{i}=\nabla^{2} \sum_{i} \varepsilon^{i} \tau_{i} .
\end{gathered}
$$

Using $\psi$ and $\tau$, the original quantities $u, v$ and $T$ are expressed as follows:

$u=\frac{\kappa}{h} \frac{\partial \psi}{\partial y}, \quad v=-\frac{\kappa}{h} \frac{\partial \psi}{\partial x}, T=h \beta_{0}\left(1+\varepsilon^{2}\right)(-y+\tau)$,

where $x$ and $y$ are non-dimensional.

The expansion method described here is also applicable to other problems, in which dependent variables are single-valued and the domain of these variables is bounded. As a simple example, the problem of one-dimensional simple harmonic oscillator is dealt with. The governing equation with regard to the velocity $v$ is

$$
v \frac{d v}{d x}+\omega^{2} x=0
$$

which, of course, can be integrated easily. But here, the above-mentioned method of expansion is followed. In (17), $x$ is upperbounded and has some maximum value, $a$ (amplitude of oscillator), as shown in Fig. 3. The situation near $x=a$ (or $x=-a$ ) in Fig. 3 is quite the same as the neighbourhood of $\beta=\beta_{0}$ in Fig. 2. Therefore $v$ and $x$ are put as

$$
x=a-\varepsilon^{2}, v=A_{1} \varepsilon+A_{2} \varepsilon^{2}+A_{3} \varepsilon^{3}+\cdots .
$$


By changing the independent variable in (17) from $x$ to $\varepsilon$, (17) becomes

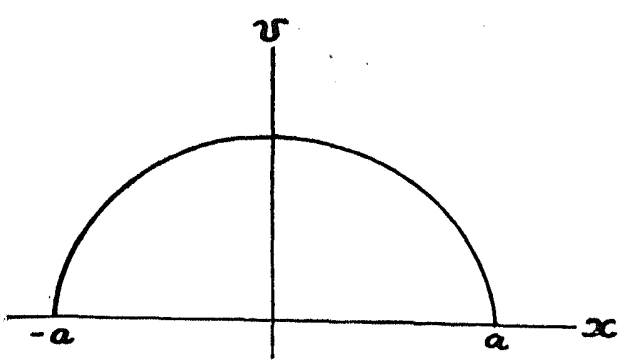

Fig. 3. Relation between velocity and displacement of a linear oscillator. $v$ is not analytic where $x= \pm a$.

$$
-\frac{v}{2 \varepsilon} \frac{d v}{d \varepsilon}+\omega^{2}\left(a-\varepsilon^{2}\right)=0
$$

Explicit determination of $A_{i}$ 's need not be mentioned, as it is quite elementary. Finally it is shown that the solution thus obtained is no other than $\frac{1}{2} v^{2}+\frac{\omega^{2}}{2} x^{2}=E$.

\$4. In this section, the sequence of equations, (14), (15) will be solved.

$[\varepsilon]:$ The first-order equations are

$$
\begin{aligned}
& \Delta^{4} \psi_{1}=R_{0} \frac{\partial \tau_{1}}{\partial x} \\
& \partial \psi_{1}=\nabla^{2} \tau_{1} . \\
& \partial: c
\end{aligned}
$$

These are concerned with the stability theory and have been exactly solved in terms of trigonometric functions (RAYLEIGH, 1916). A complete solution is

$$
\begin{gathered}
\psi_{1}=A_{1} \sin \pi y \sin \frac{\pi}{\sqrt{2}} x \\
\tau_{1}=-\frac{\sqrt{ } 2 A_{1}}{3 \pi} \sin \pi y \cos \frac{\pi}{\sqrt{ } 2} x \\
R_{0}=\frac{27}{4} \pi^{4} .
\end{gathered}
$$

This is obtained by assuming a solution in the form $e^{i m \pi x} \sin n \pi y$ and then making the value of $R$ (or the mean temperature gradient) as small as possible. Thus $R_{n}=\operatorname{Min} R(m, n)$, where $n$ is an integer. An explicit value of $A_{1}$ in (22) and (23) will be obtained in the third-order stage $\left[\varepsilon^{3}\right]$. Finally it is noted at this point that the origin of the coordinates is so chosen as to make $u=0$ at $x=0$.

$$
\begin{gathered}
\nabla^{4} \psi_{2}=R_{0} \frac{\partial \tau_{2}}{\partial x} \\
\frac{\partial \psi_{1}}{\partial y} \frac{\partial \tau_{1}}{\partial x}-\frac{\partial \psi_{1}}{\partial x} \frac{\partial \tau_{1}}{\partial y}+\frac{\partial \psi_{2}}{\partial x}=\nabla^{2} \tau_{2}
\end{gathered}
$$

Inserting (22) and (23) into (25) and (26) and solving the equations thus obtained, we get the following solution to the second-order equations:

$$
\begin{gathered}
\psi_{2}=A_{2} \sin \pi y \sin \frac{\pi}{\sqrt{2}} x \\
\tau_{2}=-\frac{\sqrt{2} A_{2}}{3 \pi} \sin \pi y \cos \frac{\pi}{\sqrt{2}} x-\frac{A_{1}{ }^{2}}{24 \pi} \sin 2 \pi y .
\end{gathered}
$$

Here again, the solution contains a new arbitrary constant $A_{2}$, which is determined explicitly in a later stage.

$\left[\varepsilon^{3}\right]: \quad \nabla^{4} \psi_{3}=R_{0} \frac{\partial \tau_{3}}{\partial x}+R_{0} \frac{\partial \tau_{1}}{\partial x}$

$$
\begin{gathered}
\frac{\partial \psi_{1}}{\partial y} \frac{\partial \tau_{2}}{\partial x}+\frac{\partial \psi_{2}}{\partial y} \frac{\partial \tau_{1}}{\partial x}-\frac{\partial \psi_{1}}{\partial x} \frac{\partial \tau_{2}}{\partial y}-\frac{\partial \psi_{2}}{\partial x} \frac{\partial \tau_{1}}{\partial y} \\
+\frac{\partial \psi_{3}}{\partial x}=\nabla^{2} \tau_{3}
\end{gathered}
$$

Inserting (22), (23), (27) and (28) into (29) and (30), we get the following equation:

$$
\begin{gathered}
\nabla^{6} \psi_{3}-R_{0} \frac{\partial^{2} \psi_{3}}{\partial x^{2}}=\frac{R_{0} \pi^{2}}{2} A_{1}\left(\frac{A_{1}^{2}}{24}-1\right) \sin \pi y \sin \frac{\pi}{\sqrt{2}} x \\
-R_{0} \frac{A_{1}^{3} \pi}{48} \sin 3 \pi y \sin \frac{\pi}{\sqrt{ } 2} x
\end{gathered}
$$

On the right hand side of (31) there are two inhomogeneous terms. The one which has the form of $\sin \pi y \sin \frac{\pi}{\sqrt{2}} x$ will generate a particular integral which does not satisfy the boundary conditions. Therefore we have to choose the value of $A_{1}$ so as to eliminate the resonant inhomogeneous term in (31), (a method used in non-linear-oscillation problems):

$$
\begin{aligned}
& R_{0} \pi^{2}-A_{1}\left(\frac{A_{1}^{2}}{24}-1\right)=0 \\
& A_{1}=\sqrt{24},-\sqrt{24}, 0
\end{aligned}
$$

Thus the determination of $A_{1}$ has been made which was impossible in the earlier stage of 
[ع]. Of the three values in $(33),-\sqrt{24}$ is discarded as it coincides with $\sqrt{24}$ by another choice of coordinates. Also $A_{1}=0$ must be discarded. This represents no other than the state of unstable rest.

$\left[\mathcal{E}^{4}\right]:$

$$
\nabla^{4} \psi_{4}=R_{0} \frac{\partial \tau_{4}}{\partial x}+R_{0} \frac{\partial \tau_{2}}{\partial x}
$$

$$
\begin{array}{r}
\frac{\partial \psi_{1}}{\partial y} \frac{\partial \tau_{3}}{\partial x}+\frac{\partial \psi_{2}}{\partial y} \frac{\partial \tau_{2}}{\partial x}+\frac{\partial \psi_{3}}{\partial y} \frac{\partial \tau_{1}}{\partial x}-\frac{\partial \psi_{1}}{\partial x} \frac{\partial \tau_{3}}{\partial y} \\
-\frac{\partial \psi_{2}}{\partial x} \frac{\partial \tau_{2}}{\partial y}-\frac{\partial \psi_{3}}{\partial x} \frac{\partial \tau_{1}}{\partial y}+\frac{\partial \psi_{4}}{\partial x}=\nabla^{2} \tau_{4}
\end{array}
$$

Rearranging the equations into a single one and eilminating the resonant inhomogeneous term, we get the following relation as in case of $\left[\varepsilon^{3}\right]$ :

$$
\begin{aligned}
& \frac{R_{0} A_{2}}{2} \pi^{2}\left(1-\frac{A_{1}^{2}}{8}\right)=0 . \\
\therefore \quad & A_{2}=0
\end{aligned}
$$

From $A_{2}=0$, it is expected that the solution in the form (12) will vanish identically, for (12) is no other than (13) having the relations: $\psi_{1}=\phi_{3}=\cdots=\tau_{1}=\tau_{3}=\cdots=0$.

Explanation of the following procedures will not be necessary. The results are just written down up to the stage of $\left[\varepsilon^{7}\right]$ :

[E]

$$
\left\{\begin{array}{l}
\psi_{1}=4.898 \sin \pi y \sin \frac{\pi}{\sqrt{2}} x \\
\tau_{1}=-0.735 \sin \pi y \cos \frac{\pi}{\sqrt{2}} x
\end{array}\right.
$$

$\left[\varepsilon^{2}\right]$

$$
\left\{\begin{array}{l}
\psi_{2}=0 \\
\tau_{2}=-0.318 \sin 2 \pi y
\end{array}\right.
$$

$$
\left\{\begin{array}{l}
\psi_{3}=0.385 \sin \pi y \sin \frac{\pi}{\sqrt{2}} x+0.019 \sin 3 \pi y \sin \frac{\pi}{\sqrt{2}} x \\
\tau_{3}=0.675 \sin \pi y \cos \frac{\pi}{\sqrt{2}} x-0.116 \sin 3 \pi y \cos \frac{\pi}{\sqrt{2}} x
\end{array}\right.
$$

$\left[\varepsilon^{4}\right]$

$$
\left\{\begin{array}{c}
\psi_{4}=-0.058 \sin 2 \pi y \sin \sqrt{2} \pi x+0.001 \sin 4 \pi y \sin \sqrt{2} \pi x \\
\tau_{4}=0.320 \sin 2 \pi y-0.025 \sin 4 \pi y+0.070 \sin 2 \pi y \cos \sqrt{2} \pi x \\
-0.011 \sin 4 \pi y \cos \sqrt{2} \pi x
\end{array}\right.
$$

[E] $\quad \varepsilon_{5}=-0.130 \sin \pi y \sin \frac{\pi}{\sqrt{2}} x+0.070 \sin \pi y \sin \frac{3 \sqrt{2}}{2} \pi x-0.006 \sin 3 \pi y \sin \frac{\pi}{\sqrt{2}} x$ $-0.001 \sin 3 \pi y \sin \frac{3 \sqrt{2}}{2} \pi x$ $\tau_{5}=-0.666 \sin \pi y \cos \frac{\pi}{\sqrt{2}} x-0.047 \sin \pi y \cos \frac{3 \sqrt{2}}{2} \pi x+0.157 \sin 3 \pi y \cos \frac{\pi}{\sqrt{2}} x$ $+0.004 \sin 3 \pi y \cos \frac{3 \sqrt{2}}{2} \pi x-0.009 \sin 5 \pi y \cos \frac{\pi}{\sqrt{2}} x$

$\left[\varepsilon^{6}\right] \quad \psi_{0}=A_{0} \sin \pi y \sin \frac{\pi}{\sqrt{2}} x+0.041 \sin 2 \pi y \sin \sqrt{2} \pi x-0.014 \sin 2 \pi y \sin 2 \sqrt{2} \pi x$ $-0.001 \sin 4 \pi y \sin \sqrt{2} \pi x$.

$\left\{\tau_{0}=-0.323 \sin 2 \pi y+0.034 \sin 4 \pi y-0.001 \sin 6 \pi y-0.1500 A_{0} \sin \pi y \cos \frac{\pi}{V} 2 x\right.$

$-0.119 \sin 2 \pi y \cos \sqrt{2} \pi x+0.005 \sin 2 \pi x \cos 2 \sqrt{2} \pi x$ $+0.017 \sin 4 \pi y \cos \sqrt{2} \pi x+0.001 \sin 4 \pi y \cos 2 \sqrt{2} \pi x$ 
$\left[\varepsilon^{7}\right] \quad \begin{aligned} \phi_{7}= & A_{7} \sin \pi y \sin \frac{\pi}{\sqrt{2}} x-0.020 \sin \pi y \sin \frac{3 \sqrt{2}}{2} \pi x+0.003 \sin 3 \pi y \sin \frac{\pi}{\sqrt{2}} x \\ \tau_{7}= & -0.130 A_{0} \sin 2 \pi y+\left(0.666-0.15\left(10 A_{7}\right) \sin \pi y \cos \frac{\pi}{\sqrt{2}} x\right. \\ & +0.061 \sin \pi y \cos \frac{3 \gamma^{\prime} 2}{2} \pi x-0.001 \sin \pi y \cos \frac{5 \sqrt{2}}{2} \pi x \\ & -0.176 \sin 3 \pi y \cos \frac{\pi}{\sqrt{2}} x-0.001 \sin 3 \pi y \cos \frac{3 \sqrt{2}}{2} \pi x \\ & +0.013 \sin 5 \pi y \cos \frac{\pi}{\sqrt{2}} x+0.001 \sin 5 \pi y \cos \frac{3 \sqrt{2}}{2} \pi x\end{aligned}$

Explicit determination of $A_{6}$ and $A_{7}$ has not yet been made, though $A_{6}$ is expected to vanish. (38) is rewritten to show the coefficient of each trigonometric function in a power series of $\varepsilon$ :

$$
\begin{aligned}
& \psi: \quad \sin \pi y \sin \frac{\pi}{\sqrt{2}} x: \quad 4.898 \varepsilon+0.385 \varepsilon^{3}-0.130 \varepsilon^{3}+A_{0} \varepsilon^{6}+A_{7} \varepsilon^{?} \\
& \sin \pi y \sin \frac{3 \pi}{\sqrt{2}} x: \quad 0.070 \varepsilon^{5}-0.020 \varepsilon^{7} \\
& \sin 2 \pi y \sin \sqrt{2} \pi x: \quad-0.058 \varepsilon^{4}+0.041 \varepsilon^{0} \\
& \sin 2 \pi y \sin 2 \sqrt{2} \pi x: \quad-0.014 \varepsilon^{6} \\
& \sin 3 \pi y \sin \frac{\pi}{\sqrt{ } 2} x: \quad 0.019 \varepsilon^{3}-0.006 \varepsilon^{5}+0.003 \varepsilon^{7} \\
& \sin 3 \pi y \sin \frac{3 \pi}{\sqrt{2}} x: \quad-0.001 \varepsilon^{3} \\
& \sin 4 \pi y \sin \sqrt{2} \pi x: \quad 0.001 \varepsilon^{4}-0.005 \varepsilon^{b} \\
& \tau: \quad \sin 2 \pi y: \quad-0.318 \varepsilon^{3}+0.320 \varepsilon^{4}-0.323 \varepsilon^{6}-0.130 A^{6} \varepsilon^{7} \\
& \sin 4 \pi y: \quad-0.025 \varepsilon^{4}+0.034 \varepsilon^{0} \\
& \sin 6 \pi y: \quad-0.001 \varepsilon^{6} \\
& \sin \pi y \cos \frac{\pi}{\sqrt{ } 2} n: \quad-0.735 \varepsilon+0.675 \varepsilon^{3}-0.666 \varepsilon^{5}-0.1500 A_{1}{ }^{6} \\
& +\left(0.666-0.1500 A_{7}\right) \varepsilon^{7} \\
& \sin \pi ! \cos \frac{3 \pi}{\sqrt{ } 2} x: \quad-0.047 \varepsilon^{5}+0.061 \varepsilon^{7} \\
& \sin \pi x \cos \frac{5 \pi}{\sqrt{2}} x: \quad-0.001 \varepsilon^{t} \\
& \sin 2 \pi y \cos \sqrt{2} \pi x: \quad 0.070 \varepsilon^{4}-0.119 \varepsilon^{6} \\
& \sin 2 \pi y \cos 2 \sqrt{ } 2 \pi x: \quad 0.005 \varepsilon^{i} \\
& \sin 3 \pi y \cos \frac{\pi}{\sqrt{ }} 2 x: \quad-0.116 \varepsilon^{3}+0.157 \varepsilon^{5}-0.176 \varepsilon^{7} \\
& \sin 3 \pi ! \cos \frac{3 \pi}{\sqrt{2}} x: \quad 0.004 \varepsilon^{5}-0.001 \varepsilon^{7} \\
& \sin 4 \pi y \cos \sqrt{2} \pi x: \quad-0.011 \varepsilon^{4}+0.017 \varepsilon^{0}
\end{aligned}
$$




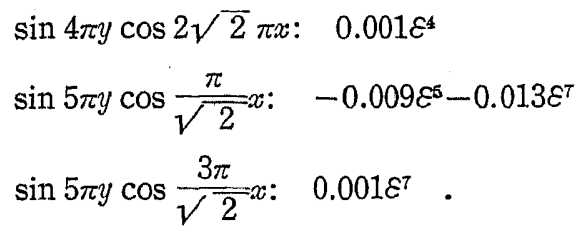

§5. The heat transport increased by convection is easily calculated from the temperature gradient at the boundaries:

$$
\begin{aligned}
& \text { Heat flow }=-k \frac{d \bar{T}}{d(h y)_{y=0}}=k \beta_{0}\left(1+\varepsilon^{2}\right)\left(1-\frac{d \bar{\tau}}{d y}\right)_{y=0} \\
& =k \beta_{0}\left(1+\varepsilon^{2}\right)\left(1+1.99 \varepsilon-1.70 \varepsilon^{2}+1.61 \varepsilon^{3}-\cdots\right)
\end{aligned}
$$

where the bars above $T$ and $\tau$ denote an average over a horizontal line. Fig. 4 shows the relation between the heat transport and the mean temperature gradient. As an illustration of convective patterns, isothermals and velocity vectors of the fluid are shown in Fig. 5, when the mean temperature gradient is $10 \%$ in excess of the critical value. The whole feature changes a little when the mean temperature gradient is $40 \%$ in excess of the critical value (Fig. 6).

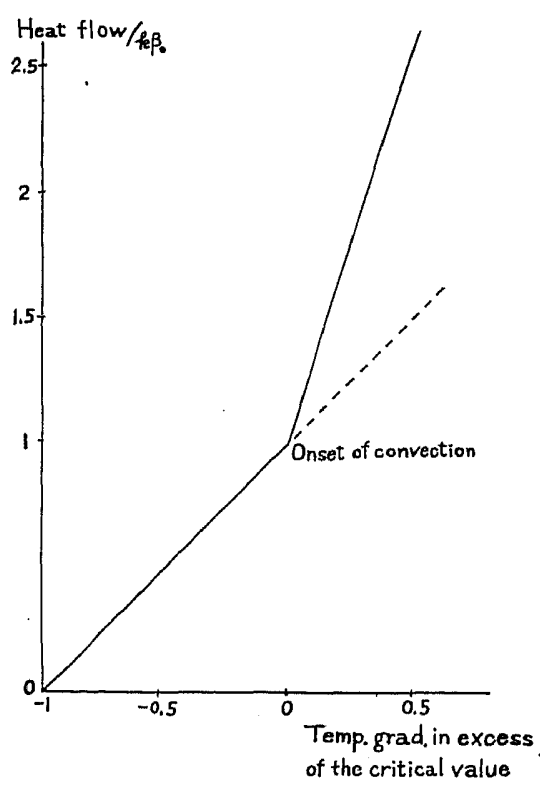

Fig. 4. Heat transport vs. temp. grad. Dashed line represents the relation due to conduction alone.

§6. Unless the fluid motion is restricted to two-dimensional, an infinite number of steady finite amplitude solutions (having different horizontal plan-forms) are obtained which formally satisfy the equations.

Another case which also gives a unique solution as in the roll case is convection

(1)

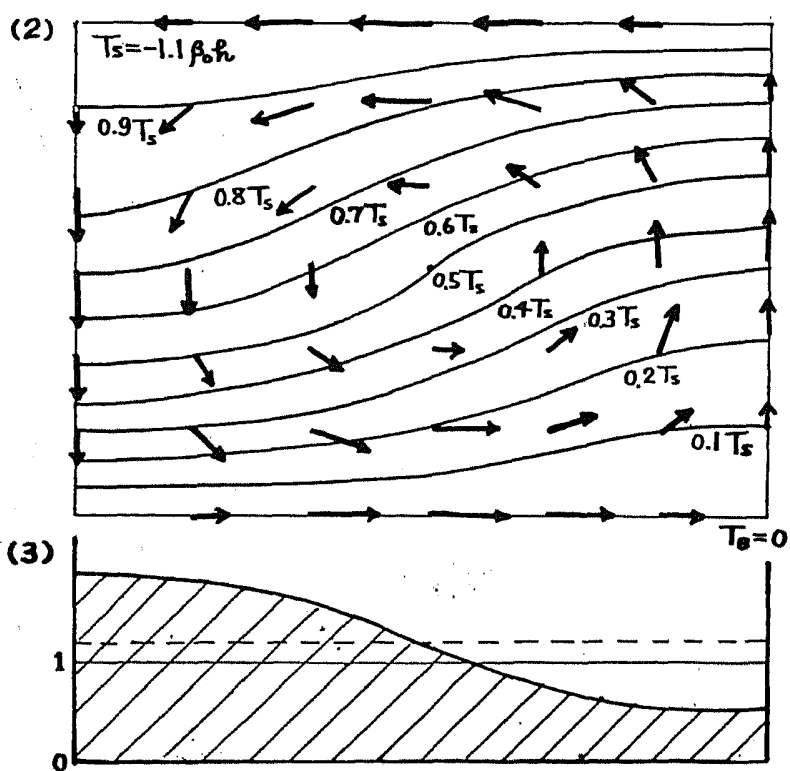

Fig. 5. Mean temp. grad. is $10 \%$ in excess of the critical value.

(1) Heat transported from above. Unit of the ordinate is the value due to conduction alone. Dashed line is the averaged value.

(2) Temperature and velocity distribution within a half of the cell. $T_{S}$ and $T_{B}$ are temperatures of the surface and the bottom respectively.

(3) Heat transported from below. 
(I)

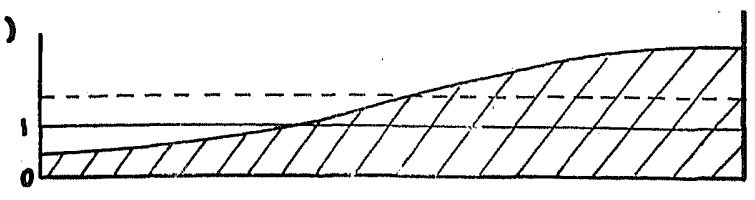

(2)

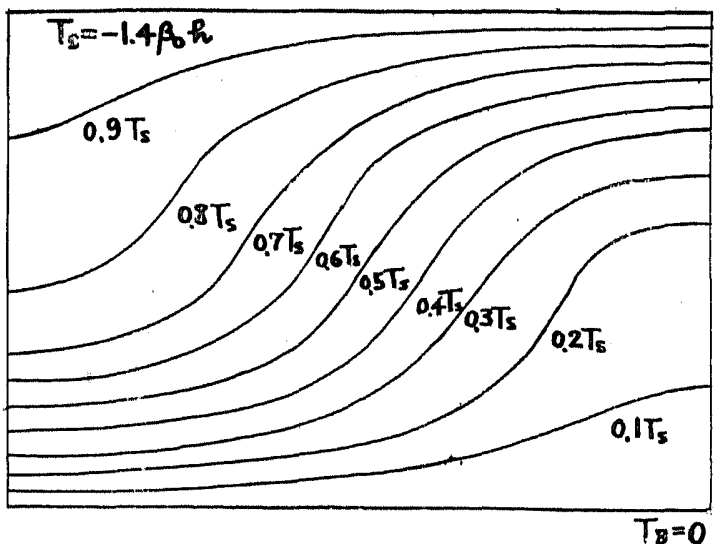

(3)

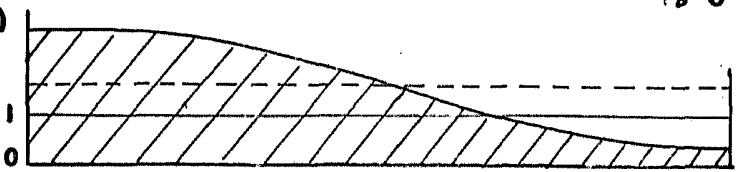

$$
-2 \overrightarrow{\beta u} \cdot \vec{r}+\vec{u} \cdot \operatorname{grad} \tau=\kappa \nabla^{2} \tau .
$$

In (42), the non-linear terms have been retained. Other equations governing the field are

$$
\begin{gathered}
\operatorname{div} \vec{u}=0, \\
0=-\operatorname{grad} p-\rho \operatorname{grad} V+\rho \nu \nabla^{2} \vec{u},
\end{gathered}
$$

where the external field $\operatorname{grad} V$ is that due to the action of gravity in a homogeneous sphere,

$$
\operatorname{grad} V=\frac{4}{3} \pi \rho_{0} \overrightarrow{G r}
$$

$G$ denotes the constant of gravitation and $\rho_{0}$ is the mean density.

By replacing $\rho$ by $\rho=\bar{\rho}(1-\alpha T)$, we get from (44)

$$
\left.\begin{array}{c}
0=-\operatorname{grad} \tilde{\omega}+r \tau \vec{r}++\nu \nabla^{2} \vec{u} \\
\tilde{\omega}=\frac{p}{\rho}-V-\frac{1}{4} \beta r\left(2 R^{2} r^{2}-r^{4}\right) \\
r=\frac{4}{3} \pi \bar{\rho} G \alpha .
\end{array}\right\}
$$

within a sphere. The following is an extension of Chandrasekhar's linearized stability theory (1952).

Following Chandrasekrhar, the sphere is taken to be a homogeneous one of radius $R$ in equilibrium under its own gravitation and with a uniform distribution of heat sources such that in the absence of conduction the temperature at each point will rise at a rate of $Q$. In the steady state, the temperature distribution, $\bar{T}(r)$, inside the sphere will be governed by

$$
\kappa \nabla^{2} \bar{T}=-Q
$$

The solution of (40) appropriate to the problem on hand is

$$
T=\beta\left(R^{2}-r^{2}\right), \quad \beta=\frac{Q}{6 \kappa} .
$$

In writing the solution in the form (41), we have assumed that $T=0$ at $r=R$; this entails no loss of generality. Let the temperature distribution in the convective state be given by $T=\bar{T}+\tau$. The equation governing $\tau$ is where

The physical quantities appearing in the above equations are non-dimensionalized as in $\$ 2$ :

$$
\tau=\beta R^{2} \tau^{\prime}, u=\frac{\kappa}{R} \overrightarrow{u^{\prime}}, \vec{r}=R \overrightarrow{r^{\prime}}
$$

After these transformations all primes will be dropped out. The following are the fundamental equations in this problem:

$$
\begin{gathered}
\operatorname{div} \vec{u}=0 \\
-2 \vec{r} \cdot \vec{u}+\vec{u} \cdot \operatorname{grad} \tau=\nabla^{2} \tau \\
0=-\operatorname{grad} \tilde{u}+\frac{C}{2} \overrightarrow{r \tau}+\nabla^{2} \vec{u}
\end{gathered}
$$

where $C \equiv \frac{2 \beta r R^{6}}{\kappa \nu}$.

$C$ corresponds to the RaYLEIGH number $R$ in $\S 2$. After eliminating $\widetilde{\omega}$ from (51), we get

$$
\nabla^{\prime}(r u)+\frac{C}{2} L^{2} \tau=0
$$


where $L^{2}=\left\{r^{2} \nabla^{2}-r \frac{\partial}{\partial r}-r \frac{\partial}{\partial r} r \frac{\partial}{\partial r}\right\}$

$$
=\frac{1}{\sin ^{2} \theta} \frac{\partial}{\partial \theta} \sin \theta \frac{\partial}{\partial \theta}+\frac{1}{\sin ^{2} \theta} \frac{\partial^{2}}{\partial \varphi^{2}}
$$

and $\vec{u}=(u, v, w)$ are the components of velocity in the spherical polar coordinates.

On the spherical boundary surface $(r=1)$, it is required

1) that the surface temperature is fixed:

$$
\tau=0
$$

which, using (52), becomes $\quad \nabla^{4}(r u)=0$,

2) that $u$ vanishes identically: $\quad r u=0, \quad(56)$ and

3) that, in case of a rigid surface, from the equation of continuity

$$
\frac{\partial}{\partial r}(r u)=0
$$

or, in case of a free surface, also from the equation of continuity

$$
\frac{\partial^{2}}{\partial r^{2}}(r u)=0 \text {. }
$$

But either of these conditions (57) and (58) makes following calculations extremely difficult. Even in case of the linear stability problem, tedious procedures of variational method are necessary. Therefore, for the sake of simplicity, we impose the following artificial conditions:

$$
\begin{aligned}
& p_{r \theta}=\rho \nu\left(\frac{\partial u}{r \partial \theta}-\frac{v}{r}+\frac{\partial v}{\partial r}\right)=-\frac{2 \rho \nu}{r} v \\
& p_{r \varphi}=\rho \nu\left(\frac{\partial u}{r \sin \theta \partial \varphi}-\frac{w}{r}+\frac{\partial w}{\partial r}\right)=-\frac{2 \rho \nu}{r} w
\end{aligned}
$$

These are reduced respectively to

$$
\begin{aligned}
& \left(\frac{\partial}{\partial r}+\frac{1}{r}\right) v=0 \\
& \left(\frac{\partial}{\partial r}+\frac{1}{r}\right) w=0 .
\end{aligned}
$$

Using the equation of continuity, (61) and (62) become

$$
\nabla^{2}(r u)=0 .
$$

From (59) and (60) it is seen that (63) is a condition between 'rigid' and 'free', which is also verified from (72) in $\$ 7$.

To sum up, the boundary conditions are (55), (56) and (63).
§7. The perturbation method used in this section is quite similar to that used in $\S 3$ and the solution is expected to be expanded in the following form

$$
\left.\begin{array}{l}
C=C_{0}\left(1+\varepsilon^{3}\right) \\
\vec{u}=\varepsilon \overrightarrow{u_{1}}+\varepsilon^{2} \vec{u}_{2}+\varepsilon^{3} \overrightarrow{u_{3}}+\cdots \\
\tau=\varepsilon \tau_{1}+\varepsilon^{2} \tau_{2}+\varepsilon^{3} \tau_{3}+\cdots
\end{array}\right\}
$$

where $C_{0}$ is the critical value of $C$ and corresponds to the critical intensity $Q_{0}$ of heat sources.

Inserting (64) into (50) and (52) we get a sequence of linear inhomogeneous equations in powers of $\varepsilon$. The following procedures are quite the same as in $\S 4$.

$[\varepsilon]$ : The first-order equation is

$$
\nabla^{\delta}\left(r u_{1}\right)=C_{0} L^{2}\left(r u_{1}\right) \text {. }
$$

Now we may assume for the solution of (65) the following form

$$
r u_{1}=W_{1}(r) Y(\theta, \varphi),
$$

where $Y$ is the spherical harmonic of the first order, as the lowest order gives $C$ the least value. The equation for $W_{1}(r)$ becomes

$$
\left[\frac{d^{2}}{d r^{2}}+\frac{2}{r} \frac{d}{d r}-\frac{2}{r^{2}}\right]^{3} W_{1}+2 C_{0} W_{1}=0 .
$$

The boundary conditions on the surface $(r=1)$ are

$$
\begin{aligned}
& \text { i) } W_{1}=0 \\
& \text { ii) }\left[\frac{d^{2}}{d r^{2}}+\frac{2}{r} \frac{d}{d r}-\frac{2}{r^{2}}\right] W_{1}=0 \\
& \text { iii) }\left[\frac{d^{2}}{d r^{2}}+\frac{2}{r} \frac{d}{d r}-\frac{2}{r^{2}}\right]^{2} W_{1}=0 .
\end{aligned}
$$

Without difficulties the solution of (67) is obtained:

$$
W_{1}=A_{1} \frac{J_{3 / 2}\left(\alpha_{1} r\right)}{\sqrt{r}},
$$

where $\alpha_{1}$ is the minimum value of $\alpha_{i}$ 's which satisfy $J_{3 / 2}\left(\alpha_{i}\right)=0$.

Thus $C_{0}$ becomes $C_{0}=\frac{\alpha_{1}{ }^{0}}{2}=4115.48$.

According to Chandrasekhar $C_{0}$ is 3091.4 in case of the free surface and 8047.1 in case of the rigid one. Therefore, in this respect too, it is seen that 63 ) represents a condition between 'rigid' and 'free'. From (66) and (71) $r u_{1}$ becomes 


$$
r u_{1}=A_{1} \frac{J_{3 / 2}\left(\alpha_{1} \gamma\right)}{\sqrt{ } r} Y_{\mathrm{t}}\left(\theta_{1} \varphi\right)
$$

Explicit value of $A_{1}$ in (73) is still unknown and is determined in the third stage $\left[\varepsilon^{3}\right]$. In addition to $A_{\downarrow}$ there are in fact two more arbitrary constants $a_{1}, b_{1}$, contained in $Y_{1}(\theta, \varphi)$, for $Y_{1}(\theta, \varphi)=\cos \theta+a_{1} \sin \theta \sin \varphi+b_{1} \sin \theta \cos \varphi$. But these will vanish when the axis $\theta=0$ is chosen artificially to be parallel to the flow at the origin. The situation, however, changes if the sphere is rotating. Later on, it is shown that the above-mentioned direction of motion is decided in regard to the rotating axis.

From $\quad-2 r u_{1}=\nabla^{2} \tau_{1}$

$\tau_{1}$ is derived as $\tau_{1}=\frac{2 A_{1}}{\alpha_{1}{ }^{2}} \frac{J_{3 / 2}\left(\alpha_{1} r\right)}{\sqrt{r}} Y_{1}(\theta, \varphi)$.

The procedure to obtain $v$ and $v$ is as follows. In general, $\vec{u}$ is expressed in the following form;

$$
\left(\begin{array}{c}
r u \\
r v \\
r w
\end{array}\right)=\left(\begin{array}{c}
\left(n F_{m, n}+r^{2} G_{n, m}\right) W_{n, m} \\
F_{n, n} \frac{\partial W_{n, m}}{\partial \theta} \\
F_{n, m} \frac{1}{\sin \theta} \frac{\partial W_{n, m}}{\partial \varphi}
\end{array}\right)+\left(\begin{array}{l}
0 \\
r \frac{L_{n, m}}{\sin \theta} \frac{\partial W_{m, n}}{\partial \varphi} \\
-r L_{n, m} \frac{\partial W_{n, m}}{\partial \theta}
\end{array}\right)
$$

where $F, G$ and $L$ are unknown functions and $W_{n, m}=r^{n} Y_{n, m}(\theta, \varphi)$. First of all, $L=0$, as easily seen from (76) and (51). Then the three unknowns are reduced to two ( $F$ and $G$ ). By an appropriate choice of a harmonic $W_{n, m}$,

these two are determined from (73); $\quad\left(F+r^{2} G\right) r=A_{1} \frac{J_{3 / 2}\left(\alpha_{1} r\right)}{\sqrt{r}}$

and from the equation of continuity; $\quad \frac{1}{r} \frac{d F}{d r}+r \frac{d G}{d r}+4 G=0$

as

$$
G=\frac{A_{1} \alpha_{1} J_{\pi / 2}\left(\alpha_{1} r\right)}{2 r^{5 / 2}}
$$

The final forms of $v_{1}$ and $w_{1}$ are

$$
\begin{gathered}
F=\frac{A_{1} J_{3 / 2}\left(\alpha_{1} r\right)}{r^{3 / 2}}-\frac{A_{1} \alpha_{1} J_{\mathfrak{B} / 2}\left(\alpha_{1} r\right)}{2 r^{1 / 2}} . \\
v_{1}=A_{1}\left[\frac{J_{3 / 2}\left(\alpha_{1} r\right)}{r^{3 / 2}}-\frac{\alpha_{1}}{2} \frac{J_{5 / 2}\left(\alpha_{1} r\right)}{r^{1 / 3}}\right] \frac{\partial Y_{1}(\theta, \varphi)}{\partial \theta}
\end{gathered}
$$

$$
w_{1}=0 \text {. }
$$

The general pattern of motion is shown in Fig. 7 .

$\left[\varepsilon^{2}\right]$ The second-order equation is

$$
\nabla^{\sigma}\left(r u_{2}\right)-C_{0} L^{2}\left(r u_{2}\right)+\frac{C_{0}}{2} L^{2}\left(\overrightarrow{u_{1}} \cdot \operatorname{grad} \tau_{1}\right)=0
$$

where $\overrightarrow{u_{1}} \cdot \operatorname{grad} \tau_{1}$ is

$$
\begin{aligned}
& 2 A_{1}^{2} J_{3 / 2}\left(\alpha_{1} r\right)\left[d-\left\{\frac{J_{3 / 2}\left(\alpha_{1} \gamma\right)}{\sqrt{r}}\right\}-\frac{J_{3 / 2}\left(\alpha_{1} r\right)}{r^{3 / 2}}\right] P_{2}(\cos \theta) \\
& +{ }_{3 \alpha_{1}{ }^{2}}^{2} \frac{A_{3} J_{3 / 2}\left(\alpha_{1} r\right)}{r^{3 / 2}}\left[2 d r\left\{\frac{J_{3 / 2}\left(\alpha_{1} r\right)}{\gamma^{\prime} r}\right\}+\frac{J_{3 / 2}\left(\alpha_{1} r\right)}{r^{3 / 2}}\right]
\end{aligned}
$$

The complete solution to the second-order equations is

$$
\begin{aligned}
& u_{2}=A_{2} \underset{r^{3 / 2}}{J_{3 / 2}\left(\alpha_{1} r\right)} \cos \theta+0.12 A_{1}{ }^{2} \frac{J_{3 / 2}\left(\beta_{1} r\right)}{r^{3 / 2}} P_{2}(\theta)+\cdots \quad \overrightarrow{u_{1}} .
\end{aligned}
$$

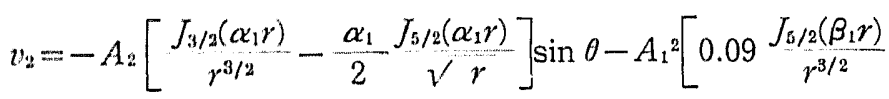

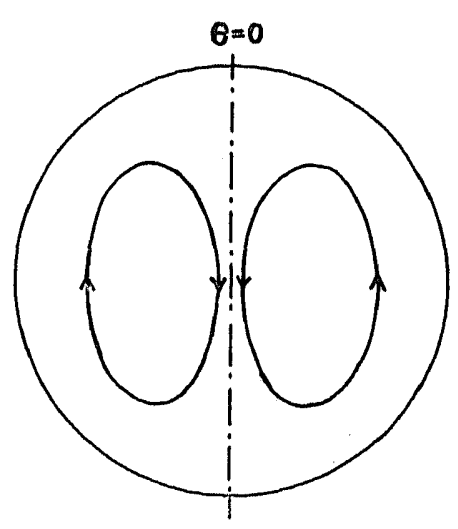

Fig. 7. General pattern of motion 


$$
\begin{aligned}
& \left.+0.03 r^{2} \frac{d}{d r}\left\{\frac{J_{5 / 2}\left(\beta_{1} r\right)}{r^{3 / 2}}\right\}\right] \sin 2 \theta+\cdots \\
w_{2}= & 0 \\
\tau_{2}= & \frac{2 A_{2}}{\alpha_{1}{ }^{2}} \frac{J_{3 / 2}\left(\alpha_{1} r\right)}{\sqrt{r}} \cos \theta+0.011 A_{1}{ }^{2} \frac{J_{5 / 2}\left(\beta_{1} r\right)}{\sqrt{r}} P_{2}(\theta)-0.0067 A_{1}{ }^{2} \frac{J_{1 / 2}(\pi r)}{\sqrt{r}} \\
& -0.0043{A_{1}{ }^{2}}^{2} \frac{J_{1 / 2}(2 \pi r)}{\sqrt{r}}+\cdots,
\end{aligned}
$$

where $J_{5} / 2\left(\beta_{n}\right)=0,(n=1,2 \cdots)$.

$\left[\varepsilon^{3}\right]$ : The third-order equation is

$$
\nabla^{3}\left(r u_{3}\right)-C_{0} L^{2}\left(r u_{3}\right)+\frac{C_{0}}{2} L^{2}\left[\nabla^{2} \tau_{1}+\vec{u}_{1} \cdot \operatorname{grad} \tau_{2}+\vec{u}_{2} \operatorname{grad} \tau_{1}\right]=0
$$

In (85), the inhomogeneous term which has the form of $\frac{J_{3 / 2}\left(\alpha_{1} \gamma\right)}{\sqrt{r}} Y_{1}(\theta, \varphi)$ will produce a secular response. Therefore, its coefficient must vanish. This leads to the determination of $A_{1}$ which, so far, has been unknown:

$$
A_{1}=7.8 \text {. }
$$

Proceeding as before, we get a solution to the third-order equations:

$$
\begin{aligned}
u_{3} & =A_{3} \frac{J_{3 / 2}\left(\alpha_{1} r\right)}{r^{3 / 2}} P_{1}(\theta)-0.75 \frac{J_{3 / 2}\left(\alpha_{1} r\right)}{r^{3 / 2}} P_{1}(\theta)+2.0 A_{2} \frac{J_{5 / 2}\left(\beta_{1} r\right)}{r^{3 / 2}} P_{2}(\theta)+48 \frac{J_{7 / 2}\left(\gamma_{1} r\right)}{r^{3 / 2}} P_{3}(\theta)+\cdots \\
\tau_{3} & =\frac{2 A_{3}}{\alpha_{1}{ }^{3}} \frac{J_{3 / 2}\left(\alpha_{1} r\right)}{r^{1 / 2}} P_{1}(\theta)-0.11 A_{2} \frac{J_{1 / 2}(\pi r)}{r^{1 / 2}}-0.063 A_{2} \frac{J_{1 / 2}(2 \pi r)}{r^{1 / 2}} \\
& -\left\{0.74 \frac{J_{3 / 2}\left(\alpha_{1} r\right)}{r^{1 / 2}}+0.65 \frac{J_{3 / 2}\left(\alpha_{2} r\right)}{r^{1 / 2}}\right\} P_{1}(\theta)+0.18 A_{2} \frac{J_{\pi / 2}\left(\beta_{1} r\right)}{r^{1 / 2}} P_{2}(\theta) \\
& +\left\{4.8 \frac{J_{7 / 2}\left(\gamma_{1} r\right)}{r^{1 / 2}}+1.6 \frac{J_{7 / 2}\left(\gamma_{2} r\right)}{r^{1 / 2}}+0.53 \frac{J_{7 / 2}\left(\gamma_{3} r\right)}{r^{1 / 2}}+0.17 \frac{J_{7 / 2}\left(r_{4} r\right)}{r^{1 / 2}}+\cdots\right\} P_{3}(\theta)+\cdots \\
v_{3} & =A_{3}\left[\frac{J_{3 / 2}\left(\alpha_{1} r\right)}{r^{3 / 2}}-\frac{\alpha_{1}}{2} \frac{J_{5 / 2}\left(\alpha_{1} r\right)}{r^{1 / 2}}\right] \frac{\partial P_{1}(\theta)}{\partial \theta}-0.75\left[\frac{J_{3 / 2}\left(\alpha_{2} r\right)}{r^{3 / 2}}+\frac{r}{2} \frac{d}{d r}\left(\frac{J_{3 / 2}\left(\alpha_{2} r\right)}{r^{3 / 2}}\right)\right] \frac{\partial P_{1}(\theta)}{\partial \theta} \\
& +1.0 A_{2} r^{2}\left[\frac{J_{5 / 2}\left(\beta_{1} r\right)}{r^{5 / 2}}+\frac{r}{3} \frac{d}{d r}\left(\frac{J_{5 / 2}\left(\beta_{1} r\right)}{r^{5 / 2}}\right)\right] \frac{\partial P_{2}(\theta)}{\partial \theta} \\
& +16 r^{3}\left[\frac{J_{5 / 2}\left(\gamma_{1} r\right)}{r^{7 / 2}}+\frac{r}{4} \frac{d}{d r}\left(\frac{J_{7 / 2}\left(\gamma_{1} r\right)}{r^{7 / 2}}\right)\right] \frac{\partial P_{3}(\theta)}{\partial \theta}+\cdots, \quad, \quad w_{3}=0
\end{aligned}
$$

where $\gamma_{i}$ are the roots of $J_{7 / 2}: J_{7 / 2}\left(\gamma_{i}\right)=0,(i=1,2 \cdots)$.

\$8. In case of a rotating sphere, calculations become more complicated, as another parameter $\Omega$ enters which is indicative of rotation. The physical quantities are expanded in double powers of $\varepsilon$ and $\Omega$. The followings are concerned only with the first order of $\varepsilon$ and $\Omega$. The chief result obtained is that the upwelling site on the surface is decided uniquely in relation to the rotating axis of the sphere.

In this case the equation of motion is, instead of (51),

$$
0=-\operatorname{grad} \widetilde{u}+\frac{C}{2} \overrightarrow{r \tau}+\nabla^{2} \vec{u}-\Omega \vec{e} \times \vec{u},
$$

where $\Omega=2 \omega R^{2} / \nu, \omega$ is the angular velocity of rotation and $\vec{e}=(\cos \theta,-\sin \theta, 0)$ is the unit vector of rotation.

First of all, we expand $C, u$, and $\tau$ in the following form,

$$
\left.\begin{array}{l}
C=\left(1+\varepsilon^{2}\right) \sum_{n=0}^{\infty} C_{n} \Omega^{n} \\
\vec{u}=\sum_{\substack{n=0 \\
m=1}}^{\infty} \varepsilon^{n} \Omega^{m} \vec{u}_{n, m}, \vec{\tau}=\sum_{\substack{n=1 \\
m=0}}^{\infty} \varepsilon^{n} \Omega^{m} \tau_{n}, m
\end{array}\right\}
$$


and then insert (89) into the governing equations (49), (50) and (88). The coefficients of $\varepsilon \Omega$ become as follows,

$$
\begin{gathered}
\operatorname{div} \overrightarrow{u_{1}, 1}=0 \\
-\overrightarrow{r r} \vec{u}_{1,1}=\nabla^{2} \tau_{1,1} \\
\vec{\nabla}^{2} \vec{u}_{1,1}-\operatorname{grad} \vec{\omega}_{1,1}+C_{0} \vec{r}\left\{\tau_{1,1}+\frac{C_{1}}{C_{0}} \tau_{1,0}\right\} \\
+\vec{u}_{1,0} \times \vec{e}=0,
\end{gathered}
$$

where $\vec{u}_{1,0}$ and $\tau_{1,0}$ are respectively previous $\overrightarrow{u_{1}}$ and $\tau_{1}$ of $\S 7$.

From (90), (91) and (92),

$$
\begin{aligned}
& \nabla^{0}\left(r u_{1,1}\right)-C_{0} L^{2}\left(r u_{1,1}\right) \\
& +C_{1} L^{2} \nabla^{2} \tau_{1,0}-\nabla^{2}\left\{\vec{r} \cdot \operatorname{rot} \operatorname{rot}\left(\vec{u}_{1,0} \times \vec{e}\right)\right\}=0 .
\end{aligned}
$$

Hitherto, the direction of the motion $\vec{u}$ at the centre of the sphere has been undetermined. But in case of rotation, it must be decided in relation to the axis of rotation. Therefore $\overrightarrow{u_{1}, 0}$ is not given by (73), (80) and (81) as before, but

$$
\overrightarrow{u_{1,0}}\left(\begin{array}{l}
u_{1,0} \\
v_{1,0} \\
w_{1,0}
\end{array}\right)=\left(\begin{array}{c}
\left(F_{1,0}+r^{2} G_{1,0}\right) r Y_{1}(\theta \cdot \varphi) \\
F_{1,0} r \frac{\partial Y_{1}}{\partial \vartheta} \\
\frac{\partial Y}{\sin \theta \partial \varphi}
\end{array}\right)
$$

where $\quad Y_{1}=\vec{a} \cos \theta+\bar{b} \sin \theta \sin \varphi+\bar{c} \sin \theta \cos \varphi$ and $\vec{a}, \vec{b}$, and $\vec{c}$ are to be decided.

Inserting (91) into (93) we get

$$
\begin{aligned}
& \nabla^{B}\left(r u_{1,1}\right)-C_{0} L^{2}\left(r u_{1,1}\right) \\
& -\nabla^{2}\left[\begin{array}{c}
J_{3 / 2}\left(\alpha_{1} r\right)\left\{\begin{array}{c}
2 C_{1} \\
V r
\end{array}\right. \\
\alpha_{1}{ }^{2}
\end{array}\right) \cos \theta+\bar{b} \sin \theta \sin \varphi \\
& +c \sin \theta \cos \varphi)+\frac{\alpha_{1}^{2}}{2}(\bar{b} \sin \theta \cos \varphi \\
& -\bar{c} \sin \theta \sin \varphi)\}]=0
\end{aligned}
$$

The inhomogeneous terms must vanish iden. tically. Therefore,

$$
C_{1} a=0, \quad \frac{2 C_{1} b}{\alpha_{1}{ }^{2}}-\frac{\alpha_{1}{ }^{2}}{2} c=0, \quad \frac{2 C_{1}}{\alpha_{1}{ }^{2}} \vec{c}+\frac{\alpha_{1}{ }^{2} \vec{b}=0}{2}
$$

i.e., $\quad c_{1}=0, \bar{b}=0, \bar{c}=0$.
This means that the fluid motion $\bar{u}_{1,0}$ is parallel to the axis of rotation at the centre of the sphere.

$$
\begin{aligned}
& \overrightarrow{u_{1}, 1} \text { and } \tau_{1,1} \text { are } \\
& u_{1,1}=\frac{J_{3 / 2}\left(\alpha_{1} \gamma\right)}{r^{3 / 2}}\left(\bar{a}_{1,1} \cos \theta+\bar{b}_{1,1} \sin \theta \sin \varphi\right. \\
& \left.+\overline{c_{1}, \mathrm{I}} \sin \theta \cos \varphi\right) \\
& \tau_{1,1}=\frac{2}{\alpha_{1}{ }^{2}} \frac{J_{3 / 2}\left(\alpha_{1} \gamma\right)}{r^{3 / 2}}\left(\bar{a}_{1,1} \cos \theta+\bar{b}_{1,1} \sin \theta \sin \varphi\right. \\
& \left.+\bar{c}_{1,1} \sin \theta \cos \varphi\right) \\
& v_{1,1}=\left\{\frac{J_{3 / 2}\left(\alpha_{1} \gamma\right)}{r^{3 / 2}}-\frac{\alpha_{1}}{2} \frac{J_{6 / 2}\left(\alpha_{1} \gamma\right)}{r^{1 / 2}}\right\}\left(-\bar{a}_{1,1} \sin \theta\right. \\
& \left.+\bar{b}_{1,1} \cos \theta \sin \varphi+{\overline{c_{1}}, 1}_{1} \cos \theta \cos \varphi\right) \\
& w_{1,1}=\left\{\frac{J_{3 / 2}\left(\alpha_{1} r\right)}{r^{3 / 2}}-\frac{\alpha_{1}}{2} \frac{J_{5 / 2}\left(\alpha_{1} r\right)}{r^{1 / 2}}\right\}\left(\bar{b}_{1,1} \cos \varphi\right. \\
& \left.-\bar{c}_{1,1} \sin \varphi\right)-0.43 \frac{J_{5 / 2}\left(\alpha_{1} r\right)}{r^{3 / 2}} \sin 2 \theta,
\end{aligned}
$$

where $\bar{a}_{1,1}, \bar{b}_{1,1}$, and $\bar{c}_{1,1}$, are all unknowns which are determined in a later stage. The general pattern of motion $-\frac{J_{5 / 2}\left(\alpha_{1} r\right)}{r^{3 / 2}} \sin 2 \theta$ is

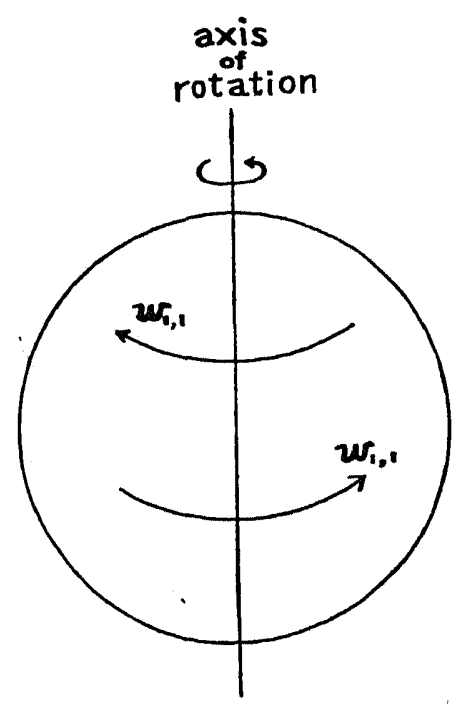

Fig. 8. General pattern of motion

$$
-\frac{J_{5 / 2}\left(\alpha_{1} r\right)}{r^{3 / 2}} \sin 20
$$

shown in Fig. 8. It is already indicative of influence of the rotation. 
§9. In the remainder of this article, a study will be made of the steady thermal convection in a two-dimensional fluid layer when it is heated uniformly from below under a simultaneous constraint of non-uniform temperature on its upper surface (inhomogeneous boundary conditions). The fundamental equations are (9) and (10). The boundary conditions are also (11) except $\tau=0,(y=0)$.

As for the non-uniform temperature distribution on the upper surface, it is assumed to have a periodicity of wave length $L$, where $L=n l, l$ is $2 \sqrt{2}$, the wave length of spontaneous cells, and $n$ is an integer. Under the assumption, it is expected that our model (Fig. 9) having a wave length $L$ corresponds

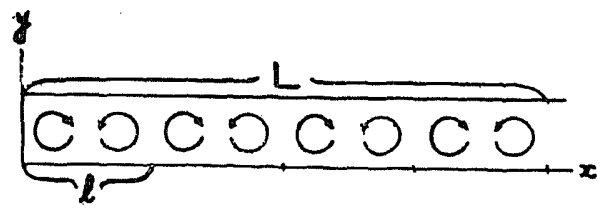

Fig. 9. Geometry of the fluid layer under investigation.

to a spherical shell. In this study $n$ is taken as 4. The non-uniformity is expressed as follows;

$$
\left.\begin{array}{l}
\tau_{t}=0, \quad(i \neq 3) \\
\tau_{3}=\sum_{n=1}^{\infty} \frac{8 \sqrt{ } 3}{2 \pi} \delta_{n} \cos \frac{2 \pi}{8 \sqrt{2}} n\left(x+\theta_{n}\right),
\end{array}\right\}(y=1)
$$

where $\delta_{n}$ 's are arbitray.

As seen from (99), the non-uniformity is solely borne on $\tau_{3}$ for the sake of mathematical technique.

$\$ 10$. In this section, calculations are carried out as in $\S 3$.

[ह]: The equations are

$$
\left\{\begin{array}{l}
\nabla^{4} \psi_{1}=R_{0} \frac{\partial \tau_{1}}{\partial x} \\
\frac{\partial \psi_{1}}{\partial x}=\nabla^{2} \tau_{1}
\end{array}\right.
$$

The boundary conditions are

$$
\frac{\partial \psi_{1}}{\partial x}=0, \quad \frac{\partial^{2} \psi_{1}}{\partial y^{2}}-\frac{\partial^{2} \psi_{1}}{\partial x^{2}}=0, \tau_{1}=0,(y=0,1) .
$$

The solution is

$$
\left\{\begin{array}{l}
\psi_{1}=A_{1} \sin \pi y \sin \frac{\pi}{\sqrt{2}}\left(x+x_{1}\right) \\
\tau_{1}=-\frac{\sqrt{2} A_{1}}{3 \pi} \sin \pi y \cos \frac{\pi}{\sqrt{2}}\left(x+x_{1}\right) .
\end{array}\right.
$$

It is to be noted here that besides $A_{1}$, an unknown phase, $x_{1}$, enters into $\phi_{1}$ and $\tau_{1}$. This comes from the consideration that the site of spontaneous convection cells is to be decided according to the surface temperature disturbance. In fact, $x_{1}$ is determined later. $\left[\varepsilon^{2}\right]$ : The equations are

$$
\left\{\begin{array}{l}
\nabla^{4} \psi_{2}=R_{0} \frac{\partial \tau_{2}}{\partial x} \\
\partial \psi_{1} \frac{\partial \tau_{1}}{\partial y}-\frac{\partial \psi_{1}}{\partial x} \frac{\partial \tau_{1}}{\partial y}+\frac{\partial \psi_{2}}{\partial x}=\nabla^{2} \tau_{2} .
\end{array}\right.
$$

The boundary conditions are

$$
\frac{\partial \psi_{2}}{\partial x}=0, \quad \frac{\partial^{y} \psi_{2}}{\partial y^{2}}-\frac{\partial^{2} \psi_{2}}{\partial x^{2}}=0, \tau_{2}=0,(y=0,1) .
$$

The solution is

$$
\left\{\begin{aligned}
\psi_{2} & =A_{2} \sin \pi y \sin \frac{\pi}{\sqrt{2}}\left(x+x_{2}\right) \\
\tau_{2} & =-\frac{\sqrt{2} A_{2}}{3 \pi} \sin \pi y \cos \frac{\pi}{\sqrt{2}}\left(x+x_{3}\right) \\
& -\frac{A_{1}^{2}}{24 \pi} \sin 2 \pi y .
\end{aligned}\right.
$$

In this stage too, a new unknown phase $x_{2}$ appears.

$\left[\varepsilon^{3}\right]:$

$$
\left\{\begin{array}{c}
\nabla^{4} \psi_{3}=R_{0} \frac{\partial \tau_{3}}{\partial x}+R_{0} \frac{\partial \tau_{1}}{\partial x} \\
\frac{\partial \psi_{1}}{\partial y} \frac{\partial \tau_{2}}{\partial x}+\frac{\partial \psi_{2}}{\partial y} \frac{\partial \tau_{1}}{\partial x}-\frac{\partial \psi_{1}}{\partial x} \frac{\partial \tau_{2}}{\partial y} \\
-\frac{\partial \psi_{2} \partial \tau_{1}}{\partial x}+\frac{\partial \psi_{3}}{\partial x}=\nabla^{2} \tau_{3}
\end{array}\right.
$$

The boundary conditions are from (99) and (11)

$$
\begin{aligned}
& \frac{\partial \psi_{3}}{\partial x}=0, \quad \frac{\partial^{2} \psi_{3}}{\partial y^{2}}-\frac{\partial^{2} \psi_{3}}{\partial x^{2}}=0, \quad(y=0,1), \\
& \tau_{3}=0, \quad(y=0) \\
& \tau_{3}=\frac{8 \sqrt{2}}{2 \pi} \sum_{n=1}^{\infty} \delta_{n} \cos \frac{2 \pi}{8 \sqrt{2}} n\left(x+\theta_{n}\right), \quad(y=1) .
\end{aligned}
$$

In the first place, (108) and (109) are cross. differentiated and then subtracted in order to get an equation governing $\psi_{3}$. 


$$
\begin{aligned}
\nabla^{6} \psi_{3} & -\frac{27}{4} \pi^{4} \frac{\partial^{2} \psi_{3}}{\partial x^{2}} \\
& =\frac{\pi^{2}}{2} A_{1} R_{0}\left[\frac{A_{1}^{2}}{24}-1\right] \sin \pi y \sin \frac{\pi}{\sqrt{2} 2^{\left(x+x_{1}\right)}} \\
& -1352 A_{1}{ }^{3} \sin 3 \pi y \sin \frac{\pi}{\sqrt{2}}\left(x+x_{1}\right) .
\end{aligned}
$$

Considering (111) we seek a solution of the form

$$
\begin{aligned}
\psi_{3} & =\sum_{n=1}^{\infty} f_{n}(y) \sin \frac{2 \pi}{8 \sqrt{2}} n\left(x+\theta_{n}\right) \\
& +0.000164 A_{1}{ }^{3} \sin \pi y \sin \frac{\pi}{\sqrt{2}}\left(x+x_{1}\right),
\end{aligned}
$$

where the last term in (113) is the particular integral of $-1352 A_{1}^{3} \sin \pi y \sin \frac{\pi}{\sqrt{2}}\left(x+x_{1}\right)$

of (112).

As the following manipulation is somewhat complicated, it will help us to rewrite the boundary conditions using $f_{n}$ 's:

$$
\begin{array}{ll}
f_{n}=0, \quad \ddot{f}_{n}=0 & (y=0,1) \\
\ldots . & \\
\dddot{f}_{n}=0, & (y=0) \\
\ldots=-R_{0} n \delta_{n} & (y=1)
\end{array}
$$

Now the problem is concerned with the functions $f_{n}$ 's. Of all these $f_{n}^{\prime}$ 's, $f_{1}$ is the most difficult one to deal with.

i) $f_{1}$. From (112) and (113) it is seen that

$$
x_{1}=\theta_{4}
$$

i.e., the phase, $x_{1}$, has been determined.

The equation governing $f_{A}$ is

$$
\begin{aligned}
& {\left[\frac{d^{2}}{d y^{2}}-\begin{array}{c}
\pi^{2} \\
2
\end{array}\right]^{3} f_{4}(y)+\left(\frac{3 \pi^{2}}{2}\right)^{3} f_{4}(y)} \\
& \quad=\frac{A_{1} R_{0}}{2} \pi^{2}\left[\frac{A_{1}^{2}}{24}-1\right] \sin \pi y .
\end{aligned}
$$

The general solution $f_{4}(y)$ of $(118)$ is the sum of the homogeneous solution $\sum_{i=1}^{0} a h_{l}(y)$ plus a particular integral $\vec{f}_{4}(y)$;

$$
\begin{aligned}
& \bar{f}_{4}(y)=-\frac{3.70 A_{1} R_{0}}{10^{2} \pi^{3}}\left[\frac{A_{1}^{2}}{24}-1\right] y \cos \pi y, \\
& \text { i.e., } \quad f_{*}(y)=\sum_{n=1}^{5} a_{i} h_{t}(y) \\
& \quad-\frac{3.70 A_{1} R_{0}}{10^{2} \pi^{3}}\left[\frac{A_{1}^{2}}{24}-1\right] y \cos \pi y,
\end{aligned}
$$

where a's have not been determined as yet, and $h_{\imath}(y)^{\prime}$ 's are the solution to the homogeneous equation of (118);

$$
\left[\frac{d^{2}}{d y^{2}}-\frac{\pi^{2}}{2}\right]^{3} h_{i}(y)+\left(\begin{array}{c}
3 \pi^{2} \\
2
\end{array}\right)^{3} h_{i}(y)=0,
$$

$\left(h_{1}, \cdots h_{6}\right)=\left(\sin \pi y, \cos \pi y, e^{(3,94+1,62 i) y,}\right.$ $\left.e^{-(3,94+1,62 i) y}, e^{(3,94-1,62 i) y}, e^{-(3,94-1,62 i) y}\right)$.

Next we have to make (120) satisfy the boundary conditions (114), (115) and (116):

$$
\begin{aligned}
\sum_{i=2}^{6} a_{i} h_{i}(0) & =0, \\
\sum_{i=2}^{6} a_{i} h_{i}(1) & =\frac{-3.70 A_{1} R_{0}}{10^{2} \pi^{3}}\left[\frac{A_{1}^{2}}{24}-1\right], \\
\sum_{i=2}^{6} a h_{i}(0) & =0, \\
\sum_{i=2}^{6} a_{i} \ddot{h_{l}}(1) & =\frac{3.70 A_{1} R_{0}}{10^{2} \pi}\left[\frac{A_{1}^{2}}{24}-1\right], \\
\sum_{i=2}^{6} a_{i} \dddot{h_{\imath}}(0) & =0, \\
\sum_{i=2}^{6} a_{i} h_{i}(1) & =\frac{-3.70 A_{1} R_{0} \pi}{10^{2}}\left[\frac{A_{1}{ }^{2}}{24}-1\right] \\
& -4 R_{0} \delta_{4},
\end{aligned}
$$

where the summation on the left-hand side is, as noted above, from 2 to 6 . This is because $h_{1}=\sin \pi y$ vanishes on the boundaries and need not be written down. In (123) there are six linear equations while the number of unknowns $a_{i}$ 's are only five. Therefore if (123) is to be consistent, all of the equations cannot be linearly independent. There must be six of such $\xi$ 's as, when multiplied by the equations (123) respectively in order, and summed up, will make the coefficients of $a$ 's vanish:

$$
\begin{gathered}
\xi_{1} h_{\iota}(0)+\xi_{2} h_{i}(1)+\xi_{3} \ddot{h}_{i}(0)+\xi_{4} \ddot{h}_{b}(1)+\xi_{0} h_{i}(0) \\
+\xi_{0} \dddot{h_{i}}(1)=0, \quad(i=2 \cdots 6) .
\end{gathered}
$$

From (124), $\xi$ 's are determined

$$
\begin{aligned}
\xi_{2}=1, \quad \xi_{3} & =\frac{10}{13 \pi^{2}}, \quad \xi_{4}=-\frac{10}{13 \pi^{2}}, \quad \xi_{5}=\frac{4}{13 \pi^{4}} \\
\xi_{0} & =\frac{4}{13 \pi^{4}},
\end{aligned}
$$

where $\xi_{1}$ is taken as 1 .

One more thing must be added. If (123) is consistent at all, these $\xi$ 's must also satisfy the following relation: 


$$
\begin{gathered}
\frac{3.70 A_{1}}{10^{2} \pi^{3}}\left[\frac{A_{1}^{2}}{24}-1\right]\left(-\xi_{2}+\pi^{2} \xi_{4}-\pi^{4} \xi_{6}\right) \\
-4 R_{0} \delta_{4} \xi_{8}=0
\end{gathered}
$$

which is derived from the inner product of the right-hand side of (123) and $\xi_{i}$ 's. (126) contains $A_{1}$ so far unknown. This means that (126) is the equation determining $A_{1}$. Inserting (1<5) into (126), the final relation determining $A_{1}$ is obtained:

$$
A_{1}\left[\begin{array}{c}
A_{1}^{2} \\
24
\end{array}-1\right]=\frac{-16}{\pi} \delta_{4} .
$$

The relation (127) is represented in Fig. 10. In some cases, for a given $\delta_{4}$ there may be three $A_{i}$ 's which formally satisfy (127), e.g., $A_{1}, A_{1}{ }^{\prime}$ and $A_{1}{ }^{\prime \prime}$ in Fig. 10 . Of these three

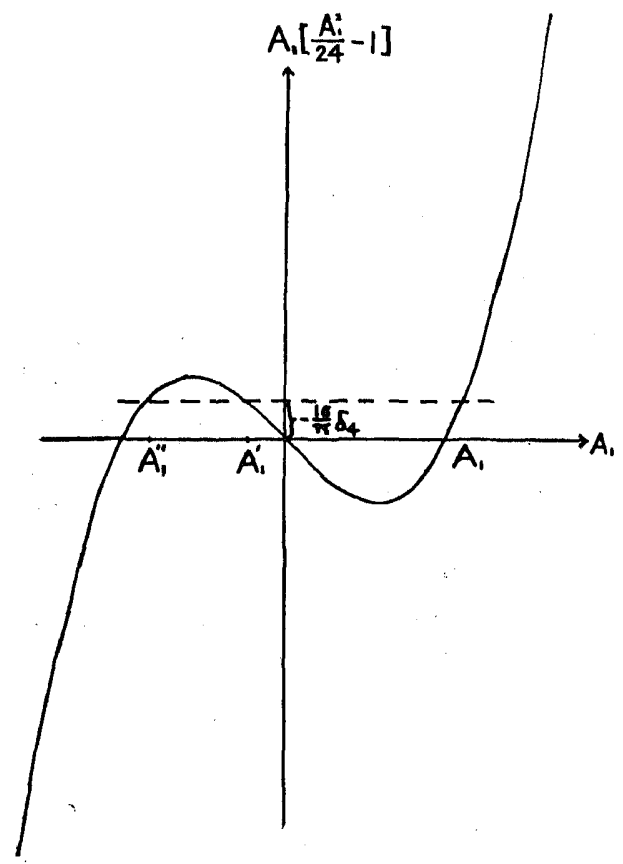

Fig. 10. Curve for deciding the amplitude of a spontaneous cell.

$A_{1}$ 's, only $A_{1}$ is the one we want, for with continuous increase of $\delta_{4}, A_{1}{ }^{\prime}$ and $A_{1}{ }^{\prime \prime}$ suddenly cease to exist. This means that $A_{1}{ }^{\prime}$ and $A_{1}{ }^{\prime \prime}$ have no physical counterparts.

The procedure to get to the relation (127) was somewhat circuitous. In another simple way, (127) is also obtainable. As in case of the GreEN's integral theorem, we first consider the homogeneous equation of (118);

$$
\begin{aligned}
\mathscr{L}[\sin \pi y] & \equiv\left[\frac{d^{2}}{d y^{2}}-\frac{\pi^{2}}{2}\right]^{3} \sin \pi y \\
& +\left(\frac{3 \pi^{2}}{2}\right)^{3} \sin \pi y=0
\end{aligned}
$$

with a solution, $\sin \pi y$ put into it. Then multiplying (128) by $f_{4}(y)$ and (118) by $\sin \pi y$ respectively, integrating them both from 0 to 1 , and finally subtracting one from the other, we get

$$
\begin{aligned}
\int_{0}^{1}\left\{f_{4}\right. & \left.\mathscr{L}[\sin \pi y]-\sin \pi y \mathscr{L}\left[f_{4}\right]\right) d y \\
= & -\frac{A_{1} R_{0}}{4} \pi^{2}\left[\frac{A_{1}^{2}}{24}-1\right] .
\end{aligned}
$$

This is the same as (127), as easily seen on integrating the left-hand side of (129) by parts. Although the present method is easier, we cannot fully rely on it, for from (129) the functional form of $f_{4}(y)$ cannot be obtained.

ii) $f_{n}(n \neq 4)$. The governing equation is

$$
\begin{aligned}
& \nabla^{6} f_{n}(y) \sin \frac{2}{8 \sqrt{2}} n\left(x+\theta_{n}\right) \\
& -\frac{27}{4} \pi^{4} f_{n}(y) \frac{d^{2}}{d x^{2}} \sin \frac{2 \pi}{8 \sqrt{2}} n\left(x+\theta_{n}\right)=0 .
\end{aligned}
$$

The boundary conditions are

$$
\begin{aligned}
& f_{n}(y)=0, \ddot{f}_{n}(y)=0, \quad(y=0,1), \dddot{f}_{n}(y)=0, \quad(y=0) \text {, } \\
& \dddot{f}_{n}(y)=-n R_{0} \delta_{n}, \quad(y=1) .
\end{aligned}
$$

We seek a solution in the form

$$
f_{n}(y)=\sum_{i=1}^{3} c_{i}^{ \pm} e^{ \pm p_{l} y^{\prime}},
$$

where $c_{i}^{ \pm}$'s are unknown constants and $p_{i}$ 's are the roots of

$$
\left(p^{2}-\frac{n^{2} \pi^{2}}{32}\right)^{3}+\frac{27 \pi^{3}}{128} n^{2}=0
$$

i.e.,

$p_{\mathrm{L}}{ }^{2}=\frac{n^{2} \pi^{2}}{32}-\frac{3 \pi^{2}}{4}\left(\frac{n^{2}}{2}\right)^{1 / 3} \equiv-s^{2}, p_{2}^{2}=\frac{n^{2} \pi^{2}}{32}$

$+\frac{3 \pi^{2}}{8}\left(\frac{n^{2}}{2}\right)^{1 / 3}(1+\sqrt{3} i) \equiv(q+r i)^{2}, p_{3}{ }^{2}=(q-r i)^{2}$.

Taking the boundary conditions (131) and (132) into account, we get for $f_{n}$ 


$$
\begin{aligned}
& f_{n}=\frac{n R_{0} \delta_{n}}{q r\left\{\left(s^{2}+q^{2}+r^{2}\right)^{2}-4 q^{2} r^{2}\right\}}\left[-q r \frac{\sin s y}{\sin s}\right. \\
& +\frac{s^{2}+q^{2}-r^{2}}{\sqrt{3\left(e^{2 q}+e^{-2 q}-2 \cos 2 r\right.}\left\{e^{q(y+1)} \cos \left(r \overline{-1}+\frac{\pi}{3}\right)\right.} \\
& +e^{-q(y+1)} \cos \left(r \overline{y-1}-\frac{\pi}{3}\right)-e^{q(y-1)} \cos \left(r \overline{y+1}+\frac{\pi}{3}\right) \\
& \left.\left.-e^{-q(y-1)} \cos \left(\overline{r y+1}-\frac{\pi}{3}\right)\right\}\right] \quad(n \neq 4) . \\
& \text { To sum up, } \psi_{3} \text { becomes as follows; } \\
& \phi_{3}=A_{3} \sin \pi y \sin \frac{\pi}{\sqrt{2}}\left(x+x_{3}\right) \\
& +0.000164 \mathrm{~A}_{1}{ }^{3} \sin 3 \pi y \sin \frac{\pi}{\sqrt{2}}\left(x+\theta_{4}\right) \\
& \quad+\sum_{n=1}^{\infty} \delta_{n} f_{n}(y) \sin \frac{2 \pi}{8 \sqrt{2}} n\left(x+\theta_{n}\right),
\end{aligned}
$$

where $f_{n}$ 's are shown in Fig. 11. Of these $f_{n}$ 's, $f_{8}$ and $f_{n}$ are larger in value than other

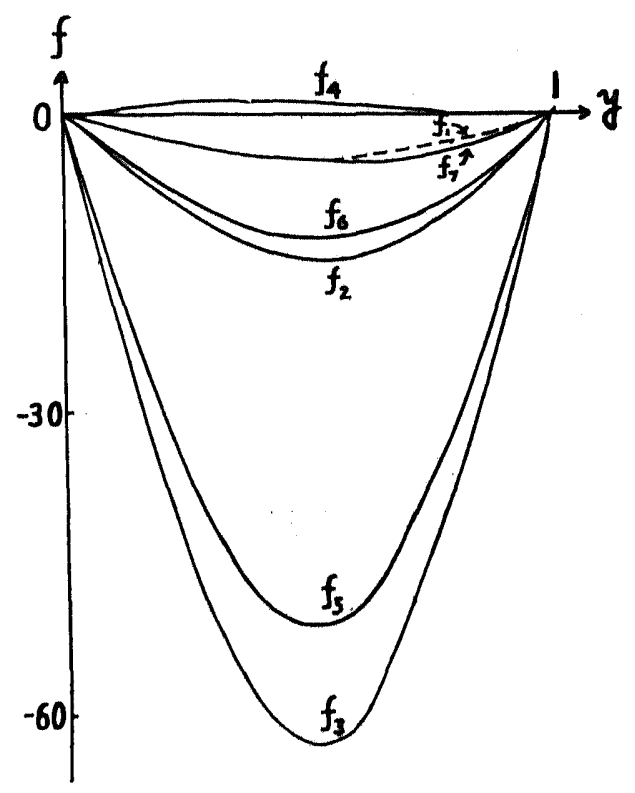

Fig. 11. $f_{i}$ 's in (48).

$f_{n}$ 's. This is due to the fact that, in case of $f_{3}$, and $f_{6}, \sin s$ in (136) is nearly zero.

$\tau_{4}$ is obtained from the auxiliary equation (108) or (109).

$$
\begin{gathered}
\tau_{3}=-A_{1} A_{2} \cos \sqrt{2 \pi} 2^{\pi}\left(\theta_{1}-x_{2}\right) \sin 2 \pi y \\
-\frac{\sqrt{2} A_{3}}{3 \pi} \sin \pi y \cos \frac{\pi}{\sqrt{2}}\left(x+x_{3}\right)
\end{gathered}
$$

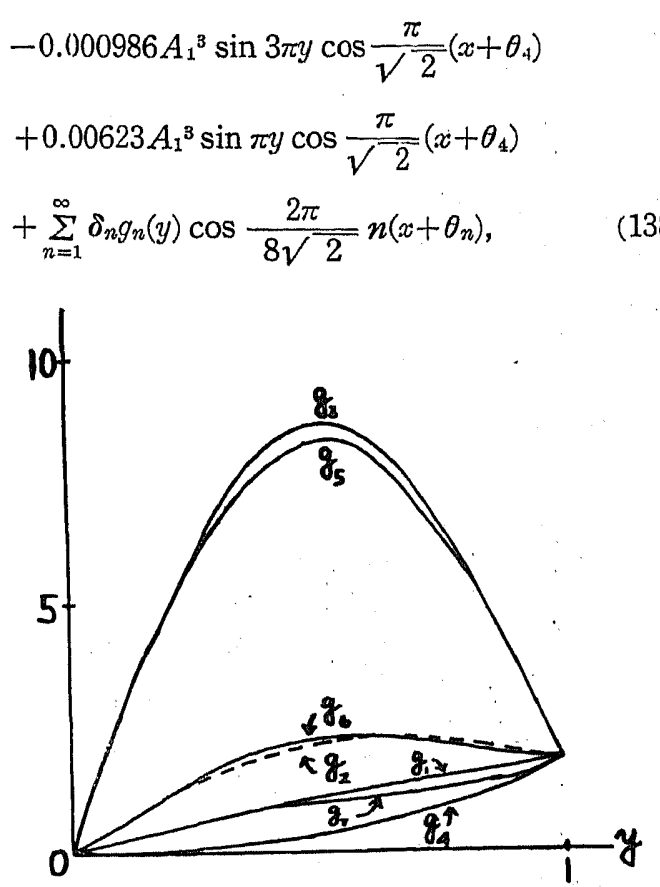

Fig. 12. $f_{i}^{\prime}$ s in (50).

where $g_{n}$ 's are shown in Fig. 12. Among these, $g_{3}$ and $g_{6}$ are large by far.

$\left[\varepsilon^{4}\right]$ : The equations are

$$
\left\{\begin{array}{l}
\nabla^{4} \psi_{4}=R_{0} \frac{\partial \tau_{4}}{\partial x}+R_{0} \frac{\partial \tau_{2}}{\partial x} \\
\frac{\partial \psi_{1}}{\partial y} \frac{\partial \tau_{3}}{\partial x}+\frac{\partial \psi_{2}}{\partial y} \frac{\partial \tau_{2}}{\partial x}+\frac{\partial \psi_{3}}{\partial y} \frac{\partial \tau_{1}}{\partial x}-\frac{\partial \psi_{1}}{\partial x} \frac{\partial \tau_{3}}{\partial y} \\
-\frac{\partial \psi_{2}}{\partial x} \frac{\partial \tau_{2}}{\partial y}-\frac{\partial \psi_{3}}{\partial x} \frac{\partial \tau_{1}}{\partial y}+\frac{\partial \psi_{4}}{\partial x}=\nabla^{2} \tau_{4} .
\end{array}\right.
$$

The boundary conditions are homogeneous. From (139) and (140) we get an equation governing $\psi_{4}$ and then the relation determin. ing $A_{2}$ :

$$
\frac{A_{2} \pi^{2}}{2}\left[\frac{A_{1}^{2}}{2}-1\right]=0 . \quad \therefore A_{2}=0 .
$$

As to the convergency, it is seen that the terms of $\left[\varepsilon^{4}\right]$ are negligible so long as $A_{1} \simeq 8$ and $\varepsilon^{2} \leq 1$. But the range of $\varepsilon$ is narrowed still more in the stage of $\left[\varepsilon^{5}\right]$, as inhomogeneous terms containing $\delta_{3}$ and $\delta_{5}$ produce a particular integral of a large value. Therefore in dealing with the $\delta_{3}$ and $\delta_{i}$ disturbances, enough consideration must be paid. 
$\S 11$. It is of interest to see how the patterns of convection are changed by the inhomogeneity constrained on the surface. This will be seen from the following several examples.

i) The temperature difference between the upper and lower surfaces is $10 \%$ in excess of the critical value. The surface temperature disturbance is in the form $\cos \frac{\pi}{\sqrt{2}} x$ and its amplitude is $10 \%$ of the temperature difference $\left(\delta_{4}=1.75\right)$. From (127), $A=-7.35$. Isothermals and stream lines in this case are shown in Fig. 13. The site of spontaneous cells is

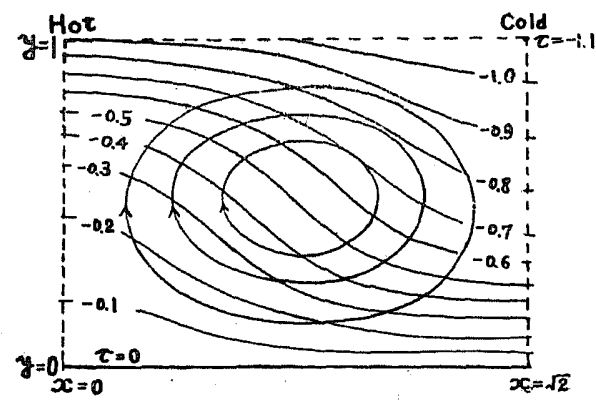

Fig. 13. Isothermals and stream lines when the temperature difference between the upper and lower surfaces is $10 \%$ in excess of the critical value. The surface temperature disturbance is of a form $\cos \frac{\pi}{\sqrt{2}} x$ and its amplitude is $10 \%$ of the temperature difference. decided entirely according to the inhomogeneity on the surface. The upwelling site is where the upper surface temperature is the highest and the sinking site is where it is the lowest. But, the pattern itself is almost the same as that with no inhomogeneity on the surface. It is to be noted here that the inhomogeneous temperature disturbance having the critical wave length, $2 \sqrt{2}$ in this case, decides once and for all the site of spontaneous convection cells, however small the disturbance may be.

ii) The temperature difference between the upper and the lower surfaces is $10 \%$ in excess of the critical value. The surface temperature disturbance is

$$
0.1 T_{a i f \jmath}\left[\cos \frac{\pi}{4 \sqrt{2}} v+\frac{1}{2} \cos \frac{\pi}{\sqrt{2}} \pi\right]
$$

Isothermals and stream lines in this case are shown in Fig. 14. It is seen from Fig. 14 that surface disturbances having much larger wave length than the critical one play very little part. Neither high nor low temperature sites on the surface seem to have considerable influence on a convective pattern. Much less can it be said from Fig. 14 that convective currents are upward where the temperature is low on the upper surface and downward where it is high. From this fact upward con-

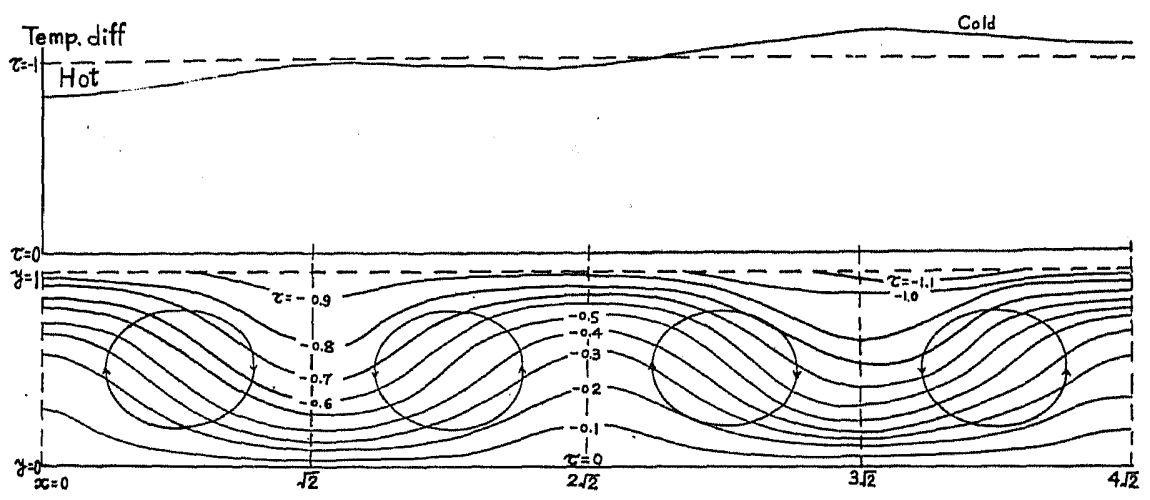

Fig. 14. Isothermals and stream lines when the temperature difference between the upper and lower surfaces is $10 \%$ in excess of the critical value. The surface temperature disturbance is $0.1 T_{\text {diff }}\left[\cos \frac{\pi}{4 \sqrt{2}} x+\frac{1}{2} \cos \frac{\pi}{\sqrt{2}} x\right]$, in olher words, the amplitude for the wave length $8 \sqrt{2}$ is $10 \%$ of the temperature difference, while that for the wave length $2 \sqrt{2}$ is half as large. 


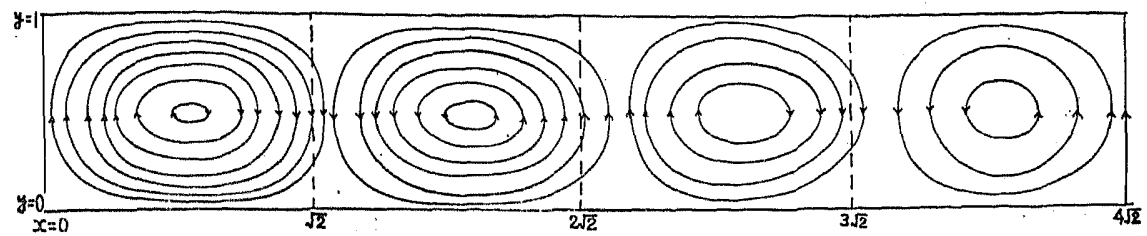

Fig. 15. Stream lines when the temperature difference between the upper and lower surfaces is only $0.3 \%$ in excess of the critical value. The surface temperature disturbance is $0.003 T_{\text {dirf }} \cos \frac{3 \pi}{4 \sqrt{2}} x+o(1) \cos \frac{\pi}{\sqrt{ } 2} x$, in other words, the amplitude for the wave length $\frac{8 \sqrt{2}}{3}$ is $0.3 \%$ of the temperature difference, while that for the wave length $2 \sqrt{2}$ is infinitesimal.

vective currents may not always be expected under the oceans where the temperature is comparatively low compared with under the continents.

iii) Surface disturbances having wave lengths close to the critical one have much influence in determining the general feature of fluid motion. In $\S 10$, these are terms concerned with $\delta_{3}$ and $\delta_{5}$.

Shown in Fig. 15 are stream lines when the temperature difference between the lower and upper surfaces is only $0.3 \%$ in excess of the critical value and the surface temperature disturbance is

$$
0.003 T_{a i s f} \cos \frac{3 \pi}{4 \sqrt{ } 2^{x+0}}+\frac{\pi}{\pi} \cos \frac{\pi}{\sqrt{2}} x \text {. }
$$

In this case, as the surface disturbance is very small, its influence is not seen very clearly. But if we compare Fig. 15 with Fig. 14 , it is seen that the disturbance of $\delta_{3}$ is very influential.

Fig. 16 shows the root mean square amplitude (in $\mathrm{km}$ ) of a spherical harmonic of order $l$ in the topographic analysis of the earth's surface after Vening Mrinesz (1951). Chandraseriar (1953) showed that the convective patterns of harmonics of orders three and four set in first when the depth of spherical shell is about half the radius of the sphere. Then he indicated the bearing of this result on the problem of convection in the earth's mantle and of the interpretation of the earth's topographic features. In this present article, it was shown that surface disturbances having much larger or smaller wave lengths than the critical one play very little part in determining the general feature of fluid motion, while those having wave lengths next to the

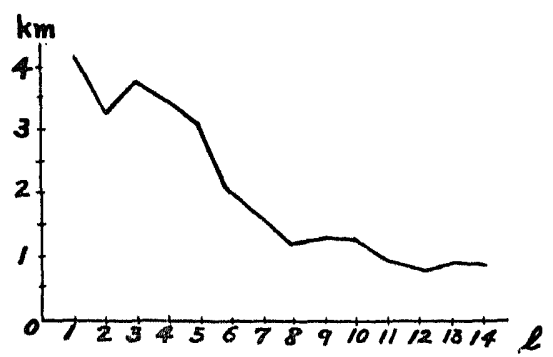

Fig. 16. Root mean square amplitude (in $\mathrm{km}$ ) of a spherical harmonic of order $l$ in the topographic analysis of the earth's surface after VEning Meinesz.

critical one are quite effective in this. This conclusion is of some interest as it is seen from Fig. 16 that the amplitude of a spherical harmonic of order 5 is considerably large compared with that of order 6 .

In conclusion, the writer wishes to express his sincere thanks to Prof. C. Tsubor and Dr. H. TAKEuchi of Tokyo University for their encouragement and support throughout the course of this study. His thanks are also due to Prof. S. Chandrasekhar of Chicago University for calling his attention to MaLkus and Veronss' study. 


\section{References}

Birch, F :

1951 "Remarkes on the structure of the mantle and its bearing upon the possibility of convection currents". Trans. Amer. Geophys. Union, 32, 533.

BLACKEETT, P.M.S.:

1956 "Lectures on rock magnetism". Jerusalem, Weitzmann Science Press.

BULLARD, E.C.:

1950 "The transfer of heat from the core of the earth". M.N.R.A.S.G.S., 6, 36.

BULlaRd, E.C.:

1954 "The earth as a planet". (Kuiper, G.P., ed.) Chapter 2. Chicago, Illinois.

BULLARD, E.C.:

1956 "Advances in geophysics". Volume 3. New York.

CHANDRASEKHAR, S.:

1952 "The thermal instability of a fluid sphere heated within". Phil. Mag. [7], 43, 1317.

ChandRASEKHAR, S.:

1953 "The onset of convection by thermal in- stability in spherical shells". Phil. Mag. [7], 44, 233.

HALES, A. L.:

1936 "Convection currents in the earth". M.N.R.A.S.G.S., 3, 372 .

HASKELL, N.A.:

1936 "The motion of a viscous fluid under a surface load". Physics, 7, 56.

MALKUS, W.V.R., and VeronIs, G.:

1958 "Finite amplitude cellular convection". J. Fluid Mech., 4, 225.

RAYLEIGH, LORD:

1916 "On convection currents in a horizontal layer of fluid, when the higher temperature is on the under side". Phil. Mag., [6], 32, 529.

VENING MEINESZ, F.A.:

1951 "A remarkable feature of the earth's topography, origin of continents and oceans". Proc. Kon. Ned. Akad. Wetensch., Series B, 54, 212, 220.

Von HERzEN, R.:

1959 "Heat flow values from the South-Eastern Pacific". Nature, 183, 882. 